\title{
Sur un système fibré lié à la suite des nombres premiers
}

\author{
Alain Costé
}

\section{SOMMAIRE}

1. Introduction

2. Le système fibré et la dynamique symbolique associée

3. La chaîne de Markov $\mathcal{P}$

4. Calcul approché de la densité stationnaire

5. Résultat des calculs sous Maple

Remerciements

Bibliographie
2000 AMS Subject Classification: Primary 37E05; Secondary 37A45

Keywords: Prime numbers, Markov chain
Nous étudions le système dynamique défini par la transformation $\Phi:[0,1] \longrightarrow] 0,1]$ où $\Phi(x)=p x-1$ si $x \in] 1 / p, 1 / q], q$ et $p$ étant deux nombres premiers consécutifs. La question de l'existence d'une mesure absolument continue invariante par $\Phi$ est reliée par un argument de chaîne de Markov à une conjecture concernant un ensemble de suites de nombres premiers. Cette hypothèse est corroborée par des simulations de type Monte-Carlo. Nous montrons que cela entraîne la stabilité statistique de $\Phi$ sur l'intervalle ]0,2/3]. En utilisant des arguments heuristiques nous définissons des versions simplifiées de l'opérateur de Perron-Frobenius associé à $\Phi$. Cela nous permet de construire à l'aide de Maple une densité de probabilité présentant une bonne adéquation expérimentale avec les histogrammes des orbites issues de constantes fondamentales.

We study the dynamical system defined by the transformation $\Phi:[0,1] \longrightarrow] 0,1]$ where $\Phi(x)=p x-1$ if $x \in] 1 / p, 1 / q], q$ and $p$ being two consecutive prime numbers. The problem of the existence of an invariant absolutely continuous measure by $\Phi$ is related via a Markov chain argument to a conjecture concerning a set of prime number sequences. This hypothesis is corroborated by Monte Carlo simulations. We prove that this implies the statistical stability of the transformation $\Phi$ on the interval ]0,2/3]. By using heuristical arguments, we define simplified versions of the Perron-Frobenius operator associated to $\Phi$. Using Maple, we construct a probability density presenting a good experimental fit with the histograms of orbits stemming from fundamental constants.

\section{INTRODUCTION}

Un système fibré est la donnée d'un ensemble I, le plus souvent un intervalle de $\mathbb{R}$ et de deux applications $\Phi: I \longrightarrow I$, (que l'on nomme plutôt transformation) et $P: I \longrightarrow \mathbb{N}$ vérifiant la propriété que la restriction de $\Phi$ à tout $P^{-1}(n)$ est injective. L'exemple classique est le système lié au développement décimal où: $I=[0,1[$, $P: x \longrightarrow[10 x]$ et $\Phi: x \longrightarrow 10 x-[10 x]$. Le système fibré lié au développement en fraction continue est défini

(c) A K Peters, Ltd. $1058-6458 / 2001 \$ 0.50$ per page Experimental Mathematics 11:3, page 383 
sur $I=] 0,1]$ par $\Phi: x \longrightarrow 1 / x-[1 / x]$ et $P: x \longrightarrow[1 / x]$. Nous renvoyons le lecteur à [Schweiger 95] pour une documentation complète sur le sujet.

Un système fibré est un système dynamique discret. Etant donnée une condition initiale $\mathrm{x}$ dans I la suite des itérés $\left(\mathrm{x}, \Phi(x), \Phi^{2}(x), \ldots\right)$ de $\mathrm{x}$ sous l'action de $\Phi$ forme une orbite dont le comportement asymptotique est le principal objet d'étude. Cette orbite engendre la suite $n \longrightarrow P_{n}(x)=P\left(\Phi^{n}(x)\right)$ que l'on appelle développement de $x$. Une suite de $\mathbb{N}$ qui est le développement d'un élément $x \in I$ est appelée suite admissible. La transmuée de la transformation $\Phi$ par l'application $P$ est le shift à droite sur l'ensemble des suites admissibles. On comprend donc l'intérêt de décrire complétement ce dernier ensemble. Dans les deux exemples précédents l'ensemble des suites admissibles est de la forme $A^{\mathbb{N}}$ où $A$ est un sous ensemble de $\mathbb{N}$. Il n'en n'est pas toujours ainsi par exemple pour le système d'Engel noté $\mathcal{E}$ [Schweiger 95]. Celui-ci est défini sur ]0,1] par

$$
\left.\Phi(x)=(k+1) x-1 \text { sur }] \frac{1}{k+1}, \frac{1}{k}\right] .
$$

Il se trouve que les suites admissibles de $\mathcal{E}$ sont exactement les suites d'entiers croissantes.

Dans ce travail nous étudions le système inspiré de $\mathcal{E}$ où l'on remplace la suite des entiers par celle des nombres premiers. Ainsi nous considérons l'application $\Phi$ définie par $x \longrightarrow p x-1$ sur l'intervalle $[1 / p, 1 / q$ [ si $q<p$ sont deux nombres premiers consécutifs. L'intervalle $] 0,1]$ est stable par $\Phi$ car d'après un théorème de Tschebychef on a toujours $p / q-1 \leq 1$ ou encore $p \leq 2 q$.

A l'instar de ce qui se passe pour $\mathcal{E}$ on s'attend à ce qu'une suite admissible soit croissante. Il n'en est rien. En fait une telle suite présente de brusques sauts suivis de descentes progressives avec une tendance marquée à revenir vers les nombres $3,5,7$ et 11 . Nous nous sommes alors intéressés aux problèmes suivants: (1) Caractérisation des suites admissibles, (2) Existence d'une densité stationnaire, (3) Ergodicité du système.

Voici maintenant exposées les grandes lignes de notre travail. Du fait que l'on ait l'inégalité plus fine $p \leq 5 / 3 q$ (si $q<p$ sont deux nombres premiers consécutifs avec $2 \leq q$ ), l'intervalle $I=] 0,2 / 3$ ] est stable par $\Phi$. De plus on voit facilement que toutes les orbites restent dans $I$ à partir d'un certain rang. Aussi du point de vue de la théorie ergodique seul le système restreint à cet intervalle est digne d'intérêt. Ce faisant on exclut simplement les suites admissibles commençant par une série de nombres 2. Par ailleurs on s'aperçoit que par rapport aux problèmes posés un certain ensemble joue un rôle central. Il s'agit de l'ensemble $\mathcal{O}$ défini comme la réunion des orbites issues des nombres $p / q-1$ (où $p<q$ sont deux premiers consécutifs avec $2 \leq q$ ). Cet ensemble est en effet très riche car le caractère premier des dénominateurs précédents fait que ces orbites sont deux à deux disjointes.

Au paragraphe 2 nous montrons qu'une suite admissible tronquée (ou $D$-suite) est une concaténation de débuts de développements de nombres $1 / p$ ( $p$ premier) avec une condition aux indices de discontinuité (appelés indices de saut). Une telle suite est donc accompagnée d'une "ombre" définie comme la suite de $\mathcal{O}$ composée de la juxtaposition des morceaux d'orbite associés aux nombres $1 / p$ mentionnés précédemment. Le dernier terme de cette "ombre" que l'on appelle résultant de la $D$-suite est une notion qui se révèle très utile au paragraphe 3 .

L'idée centrale du paragraphe 3 réside dans la constatation que l'opérateur de Perron-Frobenius $T$ associé à $\Phi$ laisse invariant le sous-espace de $L^{1}(I)$ constitué des fonctions de la forme $f=\sum_{r \in \mathcal{O}} \alpha_{r} \mathbb{1}_{0, r]}$ où $\sum\left|\alpha_{r}\right| r$ $<\infty$. La matrice $\mathcal{Z}$ de $T$ réduit à cet espace dans la base de Schauder $\left(\frac{1}{r} \mathbb{1}_{0, r]}\right)_{r \in \mathcal{O}}$ est colonne-stochastique. Nous introduisons la chaîne de Markov $\mathcal{P}$ dont l'ensemble des états est $\mathcal{O}$ et dont les probabilités de passage sont données par les coefficients de $\mathcal{Z}$. Cette chaîne est d'un grand intérêt pour l'étude des propriétés du système. A partir d'une distribution invariante $\alpha=\left[\alpha_{r}\right]_{r \in \mathcal{O}}$ de $\mathcal{P}$ on obtient une densité stationnaire de $T$ en posant $f=\sum_{r \in \mathcal{O}} \alpha_{r} / r \mathbb{1}_{0, r]}$. Comme $\mathcal{P}$ est irréductible et apériodique l'existence d'une telle distribution invariante est équivalente à l'ergodicité de la chaîne, cette propriété étant encore équivalente à la non nullité de la limite quand $n \longrightarrow \infty$ d'un quelconque des coefficients diagonaux de la matrice puissance nième de $\mathcal{Z}$. Dans le but de tester cette hypothèse nous établissons d'abord une formule exprimant le coefficient générique de la matrice $\mathcal{Z}^{n}$ par une somme portant sur les $D$-suites liées aux indices du coefficient par le biais de leurs résultants. Ainsi par exemple si $\beta_{n}$ est le coefficient diagonal de $\mathcal{Z}^{n}$ correspondant à $2 / 3$ on a

$$
\begin{aligned}
\beta_{n}=\sum\left\{\prod_{k=1}^{n} \frac{1}{p_{k}},\left(p_{1}, p_{2}, \ldots, p_{n}\right)\right. \\
\quad \text { est une } D \text {-suite de résultant } 2 / 3\} .
\end{aligned}
$$

En exploitant le fait que le résultant d'une $D$-suite est lié à la longueur du cylindre engendré par cette $D$ suite nous obtenons une expression du coefficient diagonal de $\mathcal{Z}^{n}$ comme probabilité pour qu'un nombre tiré au hasard dans un certain intervalle ait un développement 
de longueur $\mathrm{n}$ de résultant donné. Cela nous donne la possibilité de déterminer ce coefficient par la méthode de Monte Carlo. Au vu de simulations sur Maple nous nous permettons d'avancer que la limite de $\beta_{n}$ est non nulle et qu'elle est de l'ordre de 0,145 , ce qui est confirmé par le calcul approché de la densité stationnaire aux paragraphes 4 et 5 . Ainsi nous conjecturons que $\mathcal{P}$ est ergodique. Nous montrons que sous cette hypothèse la transformation $\Phi$ est statistiquement stable.

Le but du paragraphe 4 est la détermination d'une approximation la plus fine possible de la densité stationnaire $g=\sum \alpha_{r} / r \mathbb{1}_{0, r]}$ de $T$. Nous proposons deux méthodes qui sont complémentaires et dont l'élaboration est fondée sur une démarche heuristique conduisant à des approximations sans estimation effective des termes d'erreur. Nous utilisons le procédé dû à Ulam [Ulam 60] d'approximation matricielle de l'opérateur $T$ en apportant l'innovation consistant à conserver le terme reste sous forme d'un opérateur intégral.

L'opérateur $T$ est la somme d'une série d'opérateurs dont le terme général fait apparaître la suite

$$
n \longrightarrow u_{n}=\frac{p_{n}}{p_{n-1}}-1
$$

où $p_{n}$ est le nombre premier de rang n. Un entier $\mathrm{N}$ étant fixé nous définissons l'opérateur tronqué $T_{N}$ par la somme partielle d'indice $\mathrm{N}$ de la série ci-dessus et nous notons $\widetilde{T}_{N}$ l'opérateur reste $T-T_{N}$. Dans la première méthode nous nous appuyons sur le théorème des nombres premiers pour justifier le remplacement de $u_{n}$ par sa "moyenne" $1 / n$. Dans la deuxième méthode nous utilisons le modèle probabiliste de $\mathrm{H}$. Cramér [Cramér 36] pour déterminer l'espérance de $\widetilde{T}_{N} f(t)$, expression que nous adoptons comme nouvelle approximation de cet opérateur. Nous poursuivons la simplification en transformant les deux versions précédentes de $\widetilde{T}_{N}$ en un opérateur intégral $\widetilde{T}_{N}^{(i)},(i=1$ ou $i=2$ suivant la méthode utilisée). Pour cela nous faisons intervenir une fonction $G$ fournissant un "lissage" correct de la suite $n \longrightarrow p_{n}$. L'opérateur $T_{N}+\widetilde{T}_{N}^{(i)}$ laisse pratiquement stable un sous-espace $\mathcal{L}_{N}^{(i)}$ de $L^{1}(I)$ somme directe $\mathcal{E}_{N}+F^{(i)}$, où $\mathcal{E}_{N}$ est l'espace de dimension finie engendré par les $\mathbb{1}_{0, r]}, r$ parcourant la réunion des orbites issues des nombres $p_{n} / p_{n-1}-1$ (pour $n \leq N$ ) et $F^{(i)}$ est un espace de fonctions continues liées à la fonction primitive de $x \longrightarrow 1 / G(x)$. De plus l'expression de cet opérateur est suffisamment simple pour se prêter à une programmation sous Maple. Le vecteur propre de valeur propre dominante de $T_{N}+\widetilde{T}_{N}^{(i)}$ est déterminé par approximations successives. Nous définissons $g_{N}^{(i)}$ comme le nor- malisé dans $L^{1}(I)$ de ce vecteur propre. Nous observons une bonne convergence expérimentale de chaque suite $\left(g_{N}^{(i)}\right)$ vers la densité stationnaire $g$, la convergence étant plus rapide avec la seconde méthode. De plus au vu de l'expression de $g_{N}^{(i)}$ nous sommes amenés à conjecturer que $k=\lim _{t \longrightarrow 0} g(t) / \ln (t)$ existe avec $k$ de l'ordre de $-1,3$. Au dernier paragraphe nous nous intéressons au développement de quelques constantes fondamentales et nous présentons le résultat des calculs des coefficients liés à la densité stationnaire.

\section{LE SYSTÈME FIBRÉ ET LA DYNAMIQUE SYMBOLIQUE ASSOCIÉE}

La lettre $p$ désigne toujours un nombre premier différent de 1 et lorsque $p>2, p^{\sim}$ désigne le nombre premier immédiatement inférieur à $p$. Nous définissons les fonctions $P$ et $\Phi$ sur $] 0,1]$ par

$$
P(x)=\min (\{p ; 1 / x<p\}) \quad \text { et } \quad \Phi(x)=P(x) x-1 .
$$

La fonction $\Phi$ prend ses valeurs dans ]0,1] car d'après un théorème de Tschebychef on a $p<2 p^{\sim}$, (pour $p>2$ ). On peut donc itérer $\Phi$. Si l'on part de $x>2 / 3$, la suite $\Phi^{n}(x)$ commence par décroître et elle finit par prendre une valeur dans l'intervalle $I=] 0,2 / 3]$. De plus ce dernier intervalle est stable par $\Phi$ car on a l'inégalité plus fine $p \leq 5 / 3 p^{\sim}$ pour $p>2$ (cette inégalité peut se démontrer à partir de l'encadrement effectif du nombre premier de rang $n$ rappelé au début du $\oint_{4}$ ). C'est pourquoi seul le système dynamique défini par la restriction de $\Phi$ à $I$ est pris en considération. Il s'agît d'un système fibré $\sigma$-affine ([Schweiger 95]) dont la partition associée est constituée des intervalles $\left.] 1 / p, 1 / p^{\sim}\right]$ (pour $p>2$ ) et de l'intervalle $] 1 / 2,2 / 3$ ]. Aussi afin d'harmoniser les notations, nous posons $p^{\sim}=3 / 2$ (au lieu de $p^{\sim}=1$ ) lorsque $p=2$.

Nous notons pour tout $x$ de $I$ et tout entier $n \geq$ $0, P_{n}(x)=P\left(\Phi^{n}(x)\right)$.

Définition 2.1. Etant donné $x \in I$, la suite $\left[P_{n}(x)\right]_{n \geq 0}$ est appelée développement du nombre $x$ et pour tout $N \geq 1$ la suite $\left[P_{n}(x)\right]_{0 \leq n \leq N-1}$ est appelée développement d'ordre $N$ de $x$.

Proposition 2.2. Soit $x \in I$ et soit $\left(p_{k}\right)_{k \geq 0}$ son développement. On a pour tout $n \geq 0$,

$$
x=\sum_{\ell=0}^{n} \prod_{k=0}^{\ell} \frac{1}{p_{k}}+\Phi^{n+1}(x) \prod_{k=0}^{n} \frac{1}{p_{k}} .
$$


De plus

$$
x=\sum_{\ell=0}^{\infty} \prod_{k=0}^{\ell} \frac{1}{p_{k}} .
$$

Preuve: La relation $(2-1)$ est vraie pour $n=0$. En rapprochant l'égalité $(2-1)$ supposée établie pour $n$ de $\Phi^{n+1}(x)=1 / p_{n+1}\left(1+\Phi^{n+2}(x)\right)$, on voit qu'elle est encore vraie pour $n+1$. Ce qui démontre (2-1) par récurrence. La formule (2-2) en découle immédiatement.

Corollaire 2.3. Une condition nécessaire et suffisante pour que le développement de $x \in I$ soit périodique à partir d'un certain rang est que $x$ soit rationnel.

Preuve: La condition est suffisante car si $x$ est de la forme $n / m$ où $n, m \in \mathbb{N}^{*}$, alors pour tout $k, \Phi^{k}(x)$ est de la forme $n^{\prime} / m$ où $n^{\prime} \in \mathbb{N}^{*}$ et $n^{\prime}<m$. Par suite il existe $k$ et $h>0$ tels que $\Phi^{k}(x)=\Phi^{k+h}(x)$. Le développement de $x$ est donc $h$-périodique à partir du rang $k$. La condition est nécessaire. En effet si le développement de $x$ est $h$-périodique, il résulte de la proposition 2.2 que

$$
x=\sum_{\ell=0}^{h-1} \prod_{k=0}^{\ell} \frac{1}{p_{k}}\left(1-\prod_{k=0}^{h-1} \frac{1}{p_{k}}\right)^{-1} .
$$

Donc $x$ est rationnel.

Rappelons que la lettre $p$ désigne toujours un nombre premier et $p^{\sim}$ le nombre premier immédiatement inférieur à $p$ si $p>2$ et $3 / 2$ si $p=2$.

Etant donné $k \in \mathbb{N}$, nous posons

$$
r(p, k)=\Phi^{k}\left(1 / p^{\sim}\right) \quad \text { et } \quad s(p, k)=P(r(p, k)) .
$$

On a en particulier $r(p, 1)=p / p^{\sim}-1$ et $s(p, 0)=p$.

Par commodité d'écriture une suite finie ou infinie est notée $\left(p_{k}\right)_{0 \leq k<n}$ où $n \in \mathbb{N} \cup\{\infty\}$; elle est aussi notée $\left(p_{0}, \ldots, p_{n-1}\right)$ lorsque $n$ est fini.

Nous posons aussi

$$
H\left(p_{0}, \ldots, p_{n-1}\right)=\sum_{\ell=0}^{n-1} \prod_{k=0}^{\ell} \frac{1}{p_{k}}
$$

\subsection{Caractérisation des suites développements de nombres}

Définition 2.4. (Indice de bifurcation.) Soient $x, y \in$ $I, x<y$. On appelle indice de bifurcation du couple $(x, y)$ le plus petit entier $n=n(x, y)$ tel que $P_{n}(x) \neq$ $P_{n}(y)$.
Lemme 2.5. Pour tout $x, y \in I, x<y$, si $n=n(x, y)$ est l'indice de bifurcation de $(x, y)$, on $a: P_{n}(y)<P_{n}(x)$.

Preuve: On montre par récurrence la propriété $\left(\mathcal{P}_{m}\right)$ : pour tout couple $(x, y) \in I^{2}, x<y$, tel que $n(x, y)=m$, on a $P_{m}(y)<P_{m}(x)$.

$\left(\mathcal{P}_{0}\right)$ est clairement vraie. Supposons $\left(\mathcal{P}_{m}\right)$ vraie et soient $x, y \in I, x<y$, tels que $n(x, y)=m+1$, (définition 2.4). On a par hypothèse: $P_{0}(x)=P_{0}(y)=$ $p_{0}$; donc $\Phi(x)=p_{0} x-1<p_{0} y-1=\Phi(y)$. Comme l'indice de bifurcation du couple $(\Phi(x), \Phi(y))$ est égal à $m$, on peut lui appliquer la propriété $\left(\mathcal{P}_{m}\right)$, ce qui donne $P_{m+1}(y)<P_{m+1}(x)$. Donc $\left(\mathcal{P}_{m+1}\right)$ est vraie.

Définition 2.6. (Indice de saut.) Soit $S=\left(p_{k}\right)_{h \leq k<n}$, où $h \in \mathbb{N}$ et $n \in \mathbb{N} \cup\{\infty\}$, une suite de nombres premiers. On appelle indice de saut de $S$ toute valeur prise par la suite finie ou infinie $\left(k_{i}\right)_{0 \leq i<m}$ définie par récurrence par $k_{0}=h, \quad$ et pour tout $i \geq 1$ :

$k_{i}=\min \left(\left\{k ; k_{i-1}<k<n \quad\right.\right.$ et $\left.\left.\quad s\left(p_{k_{i-1}}, k-k_{i-1}\right) \neq p_{k}\right\}\right)$, si l'ensemble précédent est non vide.

Définition 2.7. (D-suite.) On dit qu'une suite $\left(p_{k}\right)_{0 \leq k<n}$ finie ou infinie de nombres premiers est une $D$-suite si pour tout couple d'indices de saut consécutifs $\left(k_{1}, k_{2}\right)$ de cette suite on a $p_{k_{2}}>s\left(p_{k_{1}}, k_{2}-k_{1}\right)$.

Exemple 2.8. $(11,2),(2,5)$ et $(11,2,11)$ sont des $D$-suites, mais $(11,2,5)$ et $(11,2,7)$ n'en sont pas. Une suite dont tous les éléments sont égaux à $p \neq 2$ est une $D$-suite. Par contre $(2,2)$ n'en est pas une.

Proposition 2.9. La suite développement d'un réel $x \in I$ est une D-suite.

Preuve: Soit $x \in I$ et soit $\left(p_{k}\right)_{k \geq 0}$ son développement. Soient $k_{1}<k_{2}$ deux indices de saut consécutifs de cette suite. En considérant $\Phi^{k_{1}}(x)$ au lieu de $x$ on peut supposer que $k_{1}=0$. Posons $y=1 / p_{0}^{\sim}$. Si $x=y$ la conclusion est immédiate car il n'y a pas de deuxième saut. Sinon $x<y$ et par définition de $k_{2}$, pour tout $k<k_{2}, p_{k}=$ $P_{k}(x)=P_{k}(y)=s\left(p_{0}, k\right)$ et $P_{k_{2}}(x) \neq P_{k_{2}}(y)$. Donc $k_{2}$ est l'indice de bifurcation de $(x, y)$. Par suite d'après le lemme $2.5, p_{k_{2}}>s\left(p_{0}, k_{2}\right)$.

Proposition 2.10. Soit $S=\left(p_{k}\right)_{0 \leq k<n}$ une D-suite. Alors pour tout $h<n$ la suite tronquée $S^{\prime}=\left(p_{k}\right)_{h \leq k<n}$ est une $D$-suite. De plus les indices de saut de $S$ supérieurs ou égaux à $h$ sont aussi des indices de saut de $S^{\prime}$. 
Preuve: Soit $k_{0}$ le plus grand des indices de saut de $S$ strictement inférieurs à $h$. Si les indices de saut de $S$ sont tous strictement inférieurs à $h$, alors $S^{\prime}$ est le développement d'ordre $n-h$ de $r\left(p_{k_{0}}, h-k_{0}\right)$. C'est donc une $D$-suite d'après la proposition 2.9. Dans le cas contraire, soit $k_{1}$ le plus petit indice de saut de $S$ minoré par $h$. Si $k_{1}=h$ la conclusion est immédiate. Autrement considérons la suite $S_{1}=\left(p_{h}, \ldots, p_{k_{1}-1}\right)$. Par définition de $k_{0}$ et de $k_{1}, S_{1}$ est le développement d'ordre $k_{1}-h$ de $r\left(p_{k_{0}}, h-k_{0}\right)$. C'est donc une $D$-suite et par conséquent l'inégalité de la définition 2.7 est vérifiée pour tout couple d'indices de saut de $S^{\prime}$ strictement inférieurs à $k_{1}$. Soit $k_{2}$ le plus grand des indices de saut de $S_{1}$. Par définition de $k_{2}$ on a:

(1) $s\left(p_{k_{2}}, k-k_{2}\right)=p_{k}$, pour tout $k$ tel que $k_{2} \leq k<k_{1}$.

De plus, par définition de $k_{0}$ et de $k_{1}$ :

(2) $s\left(p_{k_{0}}, k-k_{0}\right)=p_{k}$, pour tout $k$ tel que $k_{0} \leq k<k_{1}$. Montrons que

(3) $s\left(p_{k_{2}}, k_{1}-k_{2}\right) \leq s\left(p_{k_{0}}, k_{1}-k_{0}\right)$.

L'égalité $(2)$ avec $k=k_{2}$ implique $r\left(p_{k_{0}}, k_{2}-k_{0}\right) \leq$ $1 / p_{k_{2}}^{\sim}$. Si la relation précédente est une égalité, alors (3) est évidemment aussi une égalité. Autrement, compte tenu de (1) et (2), l'inégalité (3) est une conséquence du lemme 2.5 appliqué au couple $\left(r\left(p_{k_{0}}, k_{2}-k_{0}\right), 1 / p_{k_{2}}^{\sim}\right)$. Comme $s\left(p_{k_{0}}, k_{1}-k_{0}\right)<p_{k_{1}}$, on a donc: $s\left(p_{k_{2}}, k_{1}-k_{2}\right)<$ $p_{k_{1}}$. On en déduit que $k_{1}$ est l'indice de saut de la suite $S^{\prime}$ qui suit immédiatement $k_{2}$ et cette inégalité montre que $S^{\prime}$ est une $D$-suite. En effet, à partir de $k_{1}$ les indices de saut de $S^{\prime}$ sont les mêmes que ceux de $S$. Ce qui justifie le dernière assertion de l'énoncé.

Lemme 2.11. Soit $\left(p_{k}\right)_{0 \leq k \leq n-1}$ une D-suite finie. Alors $H\left(p_{0}, \ldots, p_{n-1}\right)<1 / p_{0}^{\sim}$.

Preuve: $\quad$ Si $n=1$ le lemme est clairement vrai. Supposons-le établi pour tout entier $m \leq n$ et soit $\left(p_{k}\right)_{0 \leq k \leq n}$ une $D$-suite de longueur $n+1$. Si 0 est le seul indice de saut de cette suite, celle-ci est le développement d'ordre $n+1$ de $1 / p_{0}^{\sim}$, donc l'inégalité est vraie. Autrement soit $k_{1}$ le deuxième indice de saut de la suite. D'après la proposition 2.10, la suite $\left(p_{k}\right)_{k_{1} \leq k \leq n-1}$ est une $D$-suite (de longueur inférieure à $n$ ). Donc par l'hypothèse de récurrence: $H\left(p_{k_{1}}, \ldots, p_{n-1}\right)<1 / p_{k_{1}}^{\sim}$.
On voit en utilisant l'égalité

$$
\begin{aligned}
H\left(p_{0}, \ldots, p_{n-1}\right)= & H\left(p_{0}, \ldots, p_{k_{1}-1}\right) \\
& +H\left(p_{k_{1}}, \ldots, p_{n-1}\right) \prod_{k=0}^{k_{1}-1} \frac{1}{p_{k}}
\end{aligned}
$$

que

$$
\begin{aligned}
H\left(p_{0}, \ldots, p_{n-1}\right)< & H\left(p_{0}, \ldots, p_{k_{1}-1}\right)+1 / p_{k_{1}}^{\sim} \prod_{k=0}^{k_{1}-1} \frac{1}{p_{k}} \\
& \leq H\left(p_{0}, \ldots, p_{k_{1}-1}, s\left(p_{0}, k_{1}\right)\right)
\end{aligned}
$$

la dernière inégalité découlant du fait que $s\left(p_{0}, k_{1}\right) \leq$ $p_{k_{1}}^{\sim}$, puisque par définition de $k_{1}, s\left(p_{0}, k_{1}\right)<p_{k_{1}}$. Or, toujours par définition de $k_{1},\left(p_{0}, \ldots, p_{k_{1}-1}, s\left(p_{0}, k_{1}\right)\right)$ est le développement d'ordre $k_{1}+1$ de $1 / p_{0}^{\sim}$. Par suite $H\left(p_{0}, \ldots, p_{k_{1}-1}, s\left(p_{0}, k_{1}\right)\right)<1 / p_{0}^{\sim}$. D'où finalement $H\left(p_{0}, \ldots, p_{n-1}\right)<1 / p_{0}^{\sim}$. Le lemme est ainsi démontré par récurrence.

Théorème 2.12. Une condition nécessaire et suffisante pour qu'une suite de nombres premiers $\left(p_{k}\right)_{k \geq 0}$ soit le développement d'un nombre réel appartenant à I est qu'elle soit une D-suite.

Preuve: La condition est nécessaire d'après la proposition 2.9. Soit $\left(p_{k}\right)_{k \geq 0}$ une $D$-suite. Montrons que cette suite est le développement du nombre $x=\lim _{n} H\left(p_{0}, \ldots, p_{n}\right)$. Il résulte du lemme 2.11 que $1 / p_{0}<x^{n} \leq 1 / p_{0}^{\sim}$. Donc $p_{0}=P_{0}(x)$. On en déduit que $\Phi(x)=p_{0} x-1$. Mais $p_{0} x-1=\lim _{n} H\left(p_{1}, \ldots, p_{n}\right)$. On voit par une récurrence sur $k$ que $p_{k}=P_{k}(x)$ pour tout $k$.

\subsection{Notion de résultant d'une $D$-suite.}

Les résultats établis dans ce paragraphe sont utiles pour la suite.

Notation. Etant donné une $D$-suite finie $S=$ $\left(p_{k}\right)_{0 \leq k<n}$, on note

$$
\begin{aligned}
& J(S)=\left\{x \in I ; P_{k}(x)=p_{k}\right. \\
& \text { pour tout k tel que } 0 \leq k<n\}
\end{aligned}
$$

et on appelle résultant de $\mathbf{S}$ le nombre $\operatorname{res}(S)=$ $r\left(p_{k_{m-1}}, n-k_{m-1}\right)$ où $k_{m-1}$ est le plus grand indice de saut de $S$. (Dans [Schweiger 95] les ensembles $J(S)$ sont appelés des cylindres).

Théorème 2.13. Soit $S=\left(p_{k}\right)_{0 \leq k<n}$ une D-suite finie. Alors on a

$$
\left.J(S)=] H\left(p_{0}, \ldots, p_{n-1}\right), H\left(p_{0}, \ldots, p_{k_{m-1}}, p_{k_{m-1}}^{\sim}\right)\right],
$$


où $k_{m-1}$ est le plus grand indice de saut de la suite $\left(p_{k}\right)_{0 \leq k<n}$.

Preuve: Soit $x \in J(S)$. On a d'après la proposition 2.2:

$$
x=H\left(p_{0}, \ldots, p_{k_{m-1}-1}\right)+\Phi^{k_{m-1}}(x) \prod_{k=0}^{k_{m-1}-1} \frac{1}{p_{k}} .
$$

Par ailleurs le développement de $\Phi^{k_{m-1}}(x)$ commence par $\left(p_{k_{m-1}}, \ldots, p_{n-1}\right)$. Donc $H\left(p_{k_{m-1}}, \ldots, p_{n-1}\right)<$ $\Phi^{k_{m-1}}(x) \leq 1 / p_{k_{m-1}}^{\sim}$. En rapprochant cette double inégalité de la relation précédente on en déduit l'encadrement souhaité de $x$.

Dans le but d'établir l'inclusion inverse montrons d'abord que

$$
H\left(p_{0}, \ldots, p_{k_{m-1}-1}, p_{k_{m-1}}^{\sim}\right) \leq 1 / p_{0}^{\sim} .
$$

$\mathrm{Du}$ fait de l'existence du saut en $k_{m-1}$, il résulte du théorème 2.12 que si l'on complète $\left(p_{0}, \ldots, p_{k_{m-1}-1}\right)$ par le développement de $1 / p_{k_{m-1}}^{\sim}$ on obtient une $D$-suite qui est le développement de $H\left(p_{0}, \ldots, p_{k_{m-1}-1}, p_{k_{m-1}}^{\sim}\right)$, ce qui prouve $(2-3)$.

Supposons d'abord que $k_{m-1}=0$. La suite $\left(p_{k}\right)_{0 \leq k<n}$ est alors le développement d'ordre $n$ de $1 / p_{0}^{\sim}$. On en déduit que $\left.] H\left(p_{0}, \ldots, p_{n-1}\right), 1 / p_{0}^{\sim}\right] \subset J(S)$.

Supposons maintenant que $k_{m-1}>0$. Soit $x \in$

$$
] H\left(p_{0}, \ldots, p_{n-1}\right), H\left(p_{0}, \ldots, p_{k_{m-1}-1}, \tilde{p}_{k_{m-1}}^{\sim}\right)\right] .
$$

D'après $(2-3)$ on a: $p_{0}=P_{0}(x)$. De plus $p_{0} x-1 \in$ ]$\left.H\left(p_{1}, \ldots, p_{n-1}\right), H\left(p_{1}, \ldots, p_{k_{m-1}-1}, p_{k_{m-1}}^{\sim}\right)\right]$. Or d'après la proposition $2.2, k_{m-1}$ est aussi le dernier indice de saut de $\left(p_{1}, \ldots, p_{n-1}\right)$. Par conséquent en supposant le théorème établi pour les suites de longueur $n-$ 1 on voit que le développement de $p_{0} x-1$ commence par $\left(p_{1}, \ldots, p_{n-1}\right)$, donc celui de $x$ commence par $\left(p_{0}, \ldots, p_{n-1}\right)$. Ce qui implique l'inclusion souhaitée pour les suites de longueur $n$. Le théorème est ainsi démontré par récurrence.

Proposition 2.14. Soit $S=\left(p_{k}\right)_{0 \leq k<n}$ une D-suite finie. Alors $J(S)$ est un intervalle de longueur $\operatorname{res}(S) \prod_{k=0}^{n-1} \frac{1}{p_{k}}$.

Preuve: Par le théorème 2.13 on sait que $J(S)$ est un intervalle de longueur

$$
\left(1 / p_{k_{m-1}}^{\sim}-H\left(p_{k_{m-1}}, \ldots, p_{n-1}\right)\right) \prod_{k=0}^{k_{m-1}-1} \frac{1}{p_{k}}
$$

où $k_{m-1}$ est le plus grand indice de saut de $S$. Mais comme $\left(p_{k_{m-1}}, \ldots, p_{n-1}\right)$ est le développement d'ordre $n-k_{m-1}$ de $1 / p_{k_{m-1}}^{\sim}$, on a d'après la proposition 2.10:

$$
\begin{aligned}
1 / p_{k_{m-1}}^{\sim}=H & \left(p_{k_{m-1}}, \ldots, p_{n-1}\right) \\
& +r\left(p_{k_{m-1}}, n-k_{m-1}\right) \prod_{k=k_{m-1}}^{n-1} \frac{1}{p_{k}} .
\end{aligned}
$$

\section{LA CHAÎNE DE MARKOV $\mathcal{P}$}

Rappelons que la lettre $p$ désigne toujours un nombre premier différent de 1 et $p^{\sim}$ désigne le nombre premier immédiatement inférieur à $p$ si $p>2$ et $3 / 2$ si $p=2$.

Le fait que les extrémités des intervalles du système fibré soient toutes (sauf une) des inverses de nombres premiers apporte ceci de particulier que les orbites finies issues de ces nombres sont deux à deux disjointes, mise à part l'exception due à l'égalité $r(2,2)=r(5,1)$. Un sousensemble de la réunion de ces orbites joue un rôle essentiel dans la suite. Il s'agit de l'ensemble $\mathcal{O}$ qui est la réunion des orbites des nombres $p / p^{\sim}-1$. Nous commençons par établir une bijection de $\mathcal{O}$ sur un ensemble de couples $(p, k)$ via l'application $(p, k) \longrightarrow r(p, k)$. Pour cela nous introduisons la fonctions $h$ suivante:

pour $p \neq 2$ et $p \neq 5$,

$$
\begin{gathered}
h(p)=\min \{k \geq 2, \text { tel qu'il existe } \mathrm{j}, 1 \leq j<k \\
\text { avec } r(p, j)=r(p, k)\}
\end{gathered}
$$

et enfin $h(2)=h(5)=2$.

Nous pouvons donc identifier $\mathcal{O}$ à l'ensemble $\{(p, k) ; p$ premier, $1 \leq k \leq h(p)-1\}$.

Nous définissons aussi la fonction j suivante:

pour $p \neq 2$ et $p \neq 5$,

$$
\begin{array}{r}
j(p)=\text { l'unique entier } \mathrm{k}, 1 \leq k<h(p) \\
\text { tel que } r(p, k)=r(p, h(p))
\end{array}
$$

et enfin $j(2)=j(5)=1$.

(En général on a pour $p>5$ :

$$
r(p, h(p)-1)=\frac{1}{p^{\sim}}
$$

donc $j(p)=1$. Le plus petit $p$ tel que $1<j(p)$ est $p=19)$.

Nous désignons par $\lambda$ la mesure de Lebesgue. Rappelons que $I=] 0,2 / 3]$.

L'application $\Phi$ définie au $\oint 2$ est non singulière, en ce sens que pour tout borélien $A$ de $I, \lambda(A)=0 \Rightarrow$ $\lambda\left(\Phi^{-1}(A)\right)=0$. On peut donc considérer l'opérateur de 


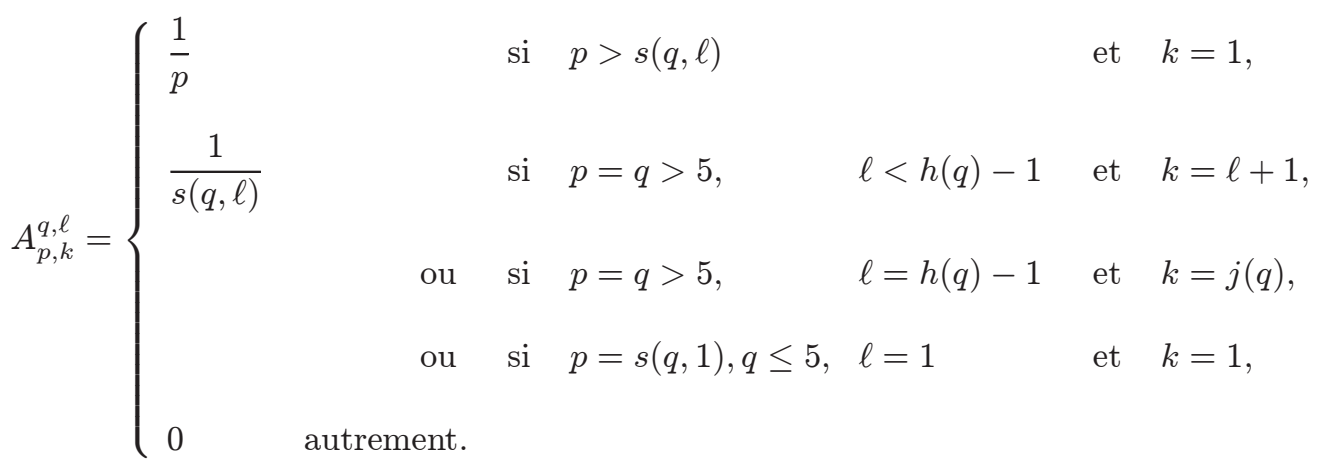

TABLE 1.

Perron-Frobenius $T$ de $L^{1}(I, \lambda)$ dans $L^{1}(I, \lambda)$ associé à $\Phi$. D'après [Schweiger 95] on a pour tout $f \in L^{1}(I, \lambda)$,

$$
T f(t)=\sum_{p} \frac{1}{p} f\left(\frac{1+t}{p}\right) \mathbb{1}_{\left.0, p / p^{\sim-1}\right]}(t),
$$

cette série étant absolument convergente pour la norme $L^{1}$. En particulier on a pour tout $s \in I$ :

$$
T\left(\mathbb{1}_{[0, s]}\right)=\frac{1}{P(s)} \mathbb{1}_{0, \Phi(s)]}+\sum_{p>P(s)} \frac{1}{p} \mathbb{1}_{0, p / p^{\sim-1]}},
$$

(pour la définition de $P(s)$ voir le début du $\S 2$ ).

Introduisons le sous-espace $\mathcal{E}$ de $L^{1}(I, \lambda)$ constitué des fonctions $f$ de la forme

$$
f=\sum_{(p, k) \in \mathcal{O}} \alpha_{p, k} \mathbb{1}_{0, r(p, k)]}
$$

où $\sum\left|\alpha_{p, k}\right| r(p, k)<\infty$. Pour simplifier l'écriture nous écrivons par la suite $e_{p, k}=\mathbb{1}_{0, r(p, k)]}$.

Le lemme suivant montre que la famille $\left(e_{p, k}\right)_{(p, k) \in \mathcal{O}}$ constitue une base de Schauder de $\mathcal{E}$.

Lemme 3.1. Soit $\left(r_{n}\right)_{n \geq 0}$ une suite injective de $\mathbb{R}_{+}^{*}$ et soit $\left(\alpha_{n}\right)_{n \geq 0}$ une suite de réels telle que $\sum\left|\alpha_{n}\right| r_{n}<\infty$. On suppose que la fonction $f=\sum \alpha_{n} \mathbb{1}_{\left.0, r_{n}\right]}$ est presque partout nulle. Alors $\alpha_{n}=0$ pour tout $n$.

Preuve: Puisque $f$ est continue à gauche, l'hypothèse implique que $f$ est identiquement nulle. Or du fait de l'injectivité de $\left(r_{n}\right)_{n \geq 0}$, on a pour tout $n: f\left(r_{n}\right)-f\left(r_{n}+\right.$ $0)=\alpha_{n}$.

On voit d'après (3-2) que $T$ laisse $\mathcal{E}$ invariant. On peut donc parler de la matrice infinie $\mathcal{A}=\left[A_{p, k}^{q, \ell}\right]_{[(p, k),(q, \ell)] \in \mathcal{O}^{2}}$ de l'opérateur $T_{\mid \mathcal{E}}$ dans la base $\left(e_{p, k}\right)_{(p, k) \in \mathcal{O}}$. Ainsi, pour tout $(q, \ell) \in \mathcal{O}$ :

$$
T e_{q, \ell}=\sum_{(p, k) \in \mathcal{O}} A_{p, k}^{q, \ell} e_{p, k} .
$$

On obtient les $A_{p, k}^{q, \ell}$ à partir de (3-2) (voir Table 1).

En particulier $A_{3,1}^{3,1}=\frac{1}{3}, \quad A_{2,1}^{5,1}=\frac{1}{2}, \quad A_{5,1}^{2,1}=\frac{1}{5}$.

Puisque $T$ est une isométrie sur $L^{1}(I, \lambda)^{+}$on a pour tout $(q, \ell) \in \mathcal{O}$ :

$$
r(q, \ell)=\sum_{(p, k) \in \mathcal{O}} r(p, k) A_{p, k}^{q, \ell} .
$$

Soit la matrice infinie

$$
\mathcal{Z}=\left[Z_{p, k}^{q, \ell}\right]_{[(p, k),(q, \ell)] \in \mathcal{O}^{2}},
$$

où

$$
Z_{p, k}^{q, \ell}=A_{(p, k)}^{(q, \ell)} \frac{r(p, k)}{r(q, \ell)} .
$$

La relation précédente montre que $\mathcal{Z}$ est colonnestochastique en ce sens que la somme des éléments de chacune de ses colonnes est égale à 1 . Cela nous permet de définir la chaîne de Markov que nous notons $\mathcal{P}$ dont $\mathcal{O}$ est l'ensemble des états et dont les $Z_{p, k}^{q, \ell}$ sont les probabilités de passage de $(q, \ell)$ à $(p, k)$.

Lemme 3.2. Pour tout $p \geq 5$, on a $p^{\sim}>s(p, 1)$.

Preuve: On a

$$
r(p, 1) \geq \frac{2}{p^{\sim}}>\frac{1}{\left(p^{\sim}\right)^{\sim}} .
$$

Donc $s(p, 1) \leq\left(p^{\sim}\right)^{\sim}$. D'où $s(p, 1)<p^{\sim}$.

Proposition 3.3. La chaîne de Markov $\mathcal{P}$ est irréductible et apériodique.

Preuve: Il s'agit de montrer que pour tout couple d'états $[(q, \ell),(p, k)]$ il existe une suite finie $\left[\left(p_{i}, k_{i}\right)\right]_{0 \leq i \leq n}$ telle que $\left(p_{0}, k_{0}\right)=(q, \ell),\left(p_{n}, k_{n}\right)=(p, k)$ et que pour tout $i$, $0 \leq i \leq n-1, A_{p_{i+1}, k_{i+1}}^{p_{i}, k_{i}} \neq 0$. 
Remarquons d'abord que tout état est accessible à partir de $(5,1)$; en effet on a pour tout $p: A_{p, 1}^{5,1} \neq 0$ et pour tout $k^{\prime}, 1 \leq k^{\prime}<h(p)-1: A_{p, k^{\prime}+1}^{p, k^{\prime}} \neq 0$.

Pour conclure il suffit d'établir que $(5,1)$ est accessible à partir de tout état $(q, \ell)$. Dans ce but choisissons $p_{1}$ tel que $p_{1}>s(q, \ell)$. Alors $A_{p_{1}, 1}^{q, \ell} \neq 0$. Si $p_{1}=5$ le résultat est évident. Autrement définissons $\left(p_{i}\right)_{2 \leq i \leq n}$ telle que $p_{n}=5$ et que $p_{i}=p_{i-1}^{\sim}$ pour tout $i, 2 \leq i \leq n$, (on rappelle que $p^{\sim}$ désigne le nombre premier immédiatement inférieur à $p$ ). Par le lemme 3.2 on a pour tout $i, 2 \leq$ $i \leq n: p_{i}>s\left(p_{i-1}, 1\right)$, d'où $A_{p_{i}, 1}^{p_{i-1}, 1} \neq 0$. La suite $\left((q, \ell),\left(p_{1}, 1\right),\left(p_{2}, 1\right), \ldots,\left(p_{n}, 1\right)\right)$ établit donc un lien entre $(q, \ell)$ et $(5,1)$.

\subsection{La chaîne $\mathcal{P}$ est-elle ergodique?}

Suivant le vocabulaire de [Feller 68] une distribution invariante de $\mathcal{P}$ est une famille $\left[\alpha_{p, k}\right]_{(p, k) \in \mathcal{O}}$ de $\mathbb{R}^{+}$telle que $\sum \alpha_{p, k}=1$ et $\mathcal{Z} \alpha=\alpha$.

La proposition suivante (qui est une conséquence directe du lemme 3.1) établit le lien entre distribution invariante de $\mathcal{P}$ et densité de probabilité stationnaire de $T$ :

Proposition 3.4. Soit $\alpha=\left[\alpha_{p, k}\right]_{(p, k) \in \mathcal{O}}$ où $\alpha_{p, k} \in$ $\mathbb{R}^{+}$et $\sum \alpha_{p, k}=1$. Une condition nécessaire et suffisante pour que $\mathcal{Z} \alpha=\alpha$, c'est-à-dire que $\alpha$ soit une distribution invariante de $\mathcal{P}$, est que la fonction $f=$ $\sum \frac{\alpha_{p, k}}{r(p, k)} \mathbb{1}_{0, r(p, k)]}(t)$ soit une densité stationnaire de $T$.

Puisque $\mathcal{P}$ est irréductible et apériodique, d'après [Feller 68, théorèmes XV.5 et XV.7] une condition nécessaire et suffisante pour qu'il existe une distribution invariante de $\mathcal{P}$ est qu'il existe au moins un état $(p, i)$ tel que $\lim _{n}{ }^{n} Z_{p, i}^{p, i} \neq 0$, (où l'on note ${ }^{n} Z_{p, i}^{q, j}$ le coefficient générique de la matrice puissance $n$-ième de $\mathcal{Z}$ ). De plus si cette limite est non nulle pour un état, elle est aussi non nulle pour tout autre état. On dit alors que la chaîne est ergodique et dans ce cas la famille $\left[\alpha_{p, k}\right]_{(p, k) \in \mathcal{O}}$ où $\alpha_{p, k}=\lim _{n}{ }^{n} Z_{p, k}^{p, k}$ constitue la seule distribution invariante de $\mathcal{P}$.

Notre but dans la suite de ce paragraphe est d'obtenir une expression des coefficients ${ }^{n} Z_{p, k}^{p, k}$ en termes de $D$ suites, ce qui donne un moyen de tester l'hypothèse d'ergodicité de $\mathcal{P}$ à l'aide de simulations numériques.

Définition 3.5. Soit $c=[(q, \ell),(p, k)] \in \mathcal{O}^{2}$. On dit qu'une suite finie $\left(p_{i}\right)_{1 \leq i \leq n}$ est $c$-compatible si la suite $\left(q, s(q, 1), \ldots, s(q, \ell-1), p_{1}, p_{2}, \ldots, p_{n}\right)$ est une $D$-suite de résultant égal à $r(p, k)$.
Proposition 3.6. Soit $c=[(q, \ell),(p, k)] \in \mathcal{O}^{2}$. Etant donné une suite finie $S=\left(p_{1}, \ldots, p_{n}\right)$, on définit $j=$ $\min \left(\left\{1 \leq i \leq n ; s(q, \ell-1+i) \neq p_{i}\right\}\right)$ si l'ensemble précédent est non vide et $j=\infty$ sinon. Alors une condition nécessaire et suffisante pour que $S$ soit c-compatible est qu'elle soit une D-suite et qu'elle satisfasse l'une ou l'autre des conditions suivantes:

(a) $j=\infty$ et $r(q, \ell+n)=r(p, k)$.

(b) $j \leq n, \quad s(q, \ell-1+j)<p_{j}$ et $\operatorname{res}(S)=r(p, k)$.

Preuve: Supposons que $S$ soit $c$-compatible. D'après la proposition 2.10, $S$ est une $D$-suite. Si de plus $j=\infty$ cela entraîne que la suite $S^{\prime}=(q, s(q, 1), \ldots, s(q, \ell-1)$, $\left.p_{1}, \ldots, p_{n}\right)$ est le développement d'ordre $n+\ell$ de $\frac{1}{q^{\sim}}$, donc $\operatorname{res}\left(S^{\prime}\right)=r(q, \ell+n)$; ainsi $S$ satisfait (a). ${ }^{2}$ i $j<\infty$ alors $j \leq n$ et le deuxième indice de saut de la suite $S^{\prime}$ définie plus haut est $j+\ell-1$; donc $s(q, \ell-1+j)<p_{j}$. De plus d'après la proposition 2.10, $j$ est un indice de saut de $S$. Donc $S$ et $S^{\prime}$ ont les mêmes résultants; ainsi $S$ satisfait (b). La réciproque est une conséquence immédiate de la proposition 2.10.

Définition 3.7. On dit qu'une suite $\left[\left(q_{i}, \ell_{i}\right)\right]_{0 \leq i \leq n}$ de $\mathcal{O}$ est une $n$-chaîne reliant $(q, \ell)$ et $(p, k)$ si $\left(q_{0}, \ell_{0}\right)=$ $(q, \ell),\left(q_{n}, \ell_{n}\right)=(p, k)$ et $A_{q_{i}, \ell_{i}}^{q_{i-1}, \ell_{i-1}} \neq 0$ pour tout i tel que $1 \leq i \leq n$.

Nous notons $C h(n,(q, \ell),(p, k))$ l'ensemble des $n$ chaînes reliant $(q, \ell)$ et $(p, k)$.

Proposition 3.8. Soit $c=[(q, \ell),(p, k)] \in \mathcal{O}^{2}$. Pour tout $n \geq 1$, l'application notée $\Delta_{n}$ qui a une $n$ chaîne $\left[\left(q_{i}, \ell_{i}\right)\right]_{0 \leq i \leq n}$ reliant $(q, \ell)$ et $(p, k)$ associe la suite $\left(\left[A_{q_{i}, \ell_{i}}^{q_{i-1}, \ell_{i-1}}\right]^{-1}\right)_{1 \leq i \leq n}$ est une bijection de $C h(n,(q, \ell),(p, k))$ sur l'ensemble des $D$-suites $c$ compatibles de longueur $n$.

Preuve: L'application $\Delta_{n}$ est injective car étant donné $(q, \ell) \in \mathcal{O}$, si pour $(p, k) \in \mathcal{O}$ on a $A_{p, k}^{q, \ell} \neq 0$, alors $(p, k)$ est déterminé par $A_{p, k}^{q, \ell}$. D'autre part lorsque $(p, k)$ parcourt l'ensemble des éléments de $\mathcal{O}$ tels que $A_{p, k}^{q, \ell} \neq 0$, le nombre $\left[A_{p, k}^{q, \ell}\right]^{-1}$ parcourt l'ensemble des $p_{1}$ tels que $p_{1} \geq s(q, \ell)$, c'est-àdire tels que $\left(q, s(q, 1), \ldots, s(q, \ell-1), p_{1}\right)$ soit une $D$ suite, dont le résultant est nécessairement $r(p, k)$. La proposition est donc vraie lorsque $n=1$. Supposons-la établie pour $n \geq 1$. Soient $c=[(q, \ell),(p, k)] \in \mathcal{O}^{2}$ et $\left[\left(q_{i}, \ell_{i}\right)\right]_{0 \leq i \leq n+1} \in C h(n+1,(q, \ell),(p, k))$. 
Posons $\quad p_{i}=\left[A_{q_{i}, \ell_{i}}^{q_{i-1}, \ell_{i-1}}\right]^{-1}, \quad 1 \leq i \leq n+1$. Montrons que la suite $S=\left(p_{i}\right)_{1 \leq i \leq n+1}$ est une $D$-suite $c$ compatible.

Discutons suivant les quatre cas où $A_{q_{1}, \ell_{1}}^{q, \ell} \neq 0$ :

(1) Si $q>5, q_{1}=q, \quad \ell<h(q)-1, \ell_{1}=\ell+1$, alors $p_{1}=s(q, \ell)=s\left(q_{1}, \ell_{1}-1\right) ; \quad$ or d'après l'hypothèse de récurrence la suite $\left(q_{1}, s\left(q_{1}, 1\right), \ldots\right.$, $\left.s\left(q_{1}, \ell_{1}-1\right), p_{2}, \ldots, p_{n+1}\right)$ est une $D$-suite de résultant $r(p, k)$. Donc $S$ est une $D$-suite $c$ compatible (définition 3.7).

(2) Si $q>5, \quad q_{1}=q, \quad \ell=h(q)-1, \ell_{1}=j(q)$, alors $p_{1}=s(q, \ell)$ et par hypothèse $\left(p_{2}, \ldots, p_{n+1}\right)$ est une $D$-suite $[(q, j(q)),(p, k)]$-compatible. Puisque $s(q, h(q)-1+i)=s(q, j(q)-1+i)$ pour tout $i \geq 1$, on voit par la proposition 3.6 que $S$ est $c$-compatible.

(3) Si $q_{1}>s(q, \ell), \ell_{1}=1$,

alors $p_{1}=q_{1}$ et par hypothèse $\left(p_{1}, p_{2}, \ldots, p_{n+1}\right)$ est une $D$-suite de résultant $r(p, k)$. Dans ce cas $\left(q, s(q, 1), \ldots, s(q, \ell-1), p_{1}, p_{2}, \ldots, p_{n+1}\right)$ est encore une $D$-suite dont l'indice $\ell$ est un indice de saut (sachant que 0 est son premier indice), de sorte que son résultant est toujours $r(p, k)$.

(4) Si $q \leq 5, q_{1}=s(q, 1), \quad \ell=1, \quad \ell_{1}=1$,

alors $p_{1}=s(q, 1)$ et par hypothèse $\left(p_{1}, p_{2}, \ldots, p_{n+1}\right)$ est une $D$-suite de résultant $r(p, k)$. Puisque $s\left(p_{1}, i\right)=s(q, i+1)$ pour tout $i \geq 1$, on voit que $\left(q, p_{1}, p_{2}, \ldots, p_{n+1}\right)$ est encore une $D$-suite de même résultant $r(p, k)$.

Montrons que inversement toute $D$-suite $S=$ $\left(p_{i}\right)_{1 \leq i \leq n+1} \quad c$-compatible est l'image par $\Delta_{n+1}$ d'une $(n+1)$-chaîne reliant $(q, \ell)$ et $(p, k)$.

Par hypothèse la suite $S^{\prime}=(q, s(q, 1), \ldots, s(q, \ell-1)$, $\left.p_{1}, p_{2}, \ldots, p_{n+1}\right)$ est une $D$-suite de résultant $r(p, k)$.

Discutons suivant les valeurs possibles de $p_{1}$ :

1. Si $p_{1}=s(q, \ell)$ et $\ell<h(q)-1$,

alors $\left(p_{2}, \ldots, p_{n+1}\right)$ est $[(q, \ell+1),(p, k)]$-compatible et d'après l'hypothèse de récurrence il existe une $n$ chaîne $\left(q_{i}, \ell_{i}\right)_{1 \leq i \leq n+1}$ telle que pour tout $2 \leq i \leq$ $n+1$,

$$
\left[A_{q_{i}, \ell_{i}}^{q_{i-1}, \ell_{i-1}}\right]^{-1}=p_{i}
$$

que $\left(q_{1}, \ell_{1}\right)=(q, \ell+1)$ et que $\left(q_{n+1}, \ell_{n+1}\right)=(p, k)$. La suite $\left(q_{i}, \ell_{i}\right)_{0 \leq i \leq n+1}$ où $\left(q_{0}, \ell_{0}\right)=(q, \ell)$ est une $(n+1)$-chaîne reliant $(q, \ell)$ et $(p, k)$ et l'image par $\Delta_{n+1}$ de cette $(n+1)$-chaîne est bien la suite $\left(p_{i}\right)_{1 \leq i \leq n+1}$.

2. Si $p_{1}=s(q, \ell)$ et $\ell=h(q)-1 \quad$ (ce qui inclus les cas où $q \leq 5$ et $\ell=1$ ),

alors $\left(p_{2}, \ldots, p_{n+1}\right)$ est $[(q, j(q)),(p, k)]$-compatible et comme précédemment on voit que la suite $\left(p_{i}\right)_{1 \leq i \leq n+1}$ est définie par une $(n+1)$-chaîne reliant $(q, \ell)$ et $(p, k)$.

3. Si $p_{1}>s(q, \ell)$,

alors d'après la proposition 2.10, la suite $\left(p_{1}, p_{2}, \ldots, p_{n+1}\right) \quad$ est une $D$-suite de même résultant que la suite $S^{\prime}$. Donc $\left(p_{2}, \ldots, p_{n+1}\right)$ est $\left[\left(p_{1}, 1\right),(p, k)\right]$-compatible. Par hypothèse de récurrence il existe une $n$-chaîne $\left(q_{i}, \ell_{i}\right)_{1 \leq i \leq n+1}$ telle que pour tout $2 \leq i \leq n+1$,

$$
\left[A_{q_{i}, \ell_{i}}^{q_{i-1}, \ell_{i-1}}\right]^{-1}=p_{i},
$$

que $\left(q_{1}, \ell_{1}\right)=\left(p_{1}, 1\right)$ et que $\left(q_{n+1}, \ell_{n+1}\right)=(p, k)$. Comme $A_{p_{1}, 1}^{q, \ell}=\frac{1}{p_{1}}$, on obtient une $(n+1)$-chaîne reliant $(q, \ell)$ et $(p, k)$ en prolongeant la précédente par $\left(q_{0}, \ell_{0}\right)=(q, \ell)$; de plus l'image par $\Delta_{n+1}$ de cette $(n+1)$-chaîne est bien la suite $\left(p_{i}\right)_{1 \leq i \leq n+1}$.

La proposition est donc démontrée par récurrence.

Le résultat suivant est une conséquence directe de ce qui précède:

Proposition 3.9. Soit $[(q, \ell),(p, k)] \in \mathcal{O}^{2}$. Alors pour tout $n \geq 1$, si ${ }^{n} A_{p, k}^{q, \ell}$ désigne le coefficient générique de la puissance $n$-ième de la matrice $\mathcal{A}$, on a

$$
\begin{aligned}
{ }^{n} A_{p, k}^{q, \ell} & =\sum\left\{\prod_{k=1}^{n} \frac{1}{p_{k}} ;\left(p_{1}, p_{2}, \ldots, p_{n}\right)\right. \\
& \text { est une D-suite }[(q, \ell),(p, k)] \text {-compatible }\} .
\end{aligned}
$$

Nous avons vu plus haut que la question de l'ergodicité de la chaîne $\mathcal{P}$ se ramène à la non nullité de $\lim _{n}{ }^{n} Z_{5,1}^{5,1}$. Or on a ${ }^{n} Z_{5,1}^{5,1}={ }^{n} A_{5,1}^{5,1}$. De plus une condition nécessaire et suffisante pour qu'une suite $\left(p_{1}, p_{2}, \ldots, p_{n}\right)$ soit $[(5,1),(5,1)]$-compatible est qu'elle soit une $D$-suite de résultant $\frac{2}{3}$. Ainsi d'après la proposition 3.9 nous pouvons écrire:

$$
{ }^{n} Z_{5,1}^{5,1}=\sum\left\{\prod_{k=1}^{n} \frac{1}{p_{k}},\left(p_{1}, p_{2}, \ldots, p_{n}\right)\right.
$$

est une $D$-suite de résultant $2 / 3\}$. 
Les valeurs approchées de ${ }^{n} Z_{5,1}^{5,1}$ pour $n=2,3$, 4 , 5, obtenues par calcul direct sont respectivement $0,253 \ldots 0,183 \ldots, 0,171 \ldots, 0,160 \ldots$

Le nombre de termes dans la somme ci-dessus est asymptotiquement d'ordre plus grand que $\gamma^{n^{2}}$ pour un certain $\gamma>1$. Le calcul des ${ }^{n} Z_{5,1}^{5,1}$ est tout de même possible par le procédé de Monte Carlo. Pour cela on nous nous appuyons sur les propositions suivantes:

Proposition 3.10. Soit $n \geq 1$. Si $x$ est pris au hasard dans I suivant la loi uniforme, la probabilité pour que la D-suite formée des $n$ premiers nombres de son développement soit de résultant $2 / 3$ est égale à ${ }^{n} Z_{5,1}^{5,1}$.

Preuve: Suivant les notations du paragraphe 2.2, l'ensemble des $x \in I$ ayant la propriété de l'énoncé est égal à la réunion disjointe des $J(S)$ où $S$ parcourt l'ensemble des $D$-suites de longueur $n$ et de résultant $2 / 3$. Le résultat découle alors de la proposition 3.9 et de la proposition 2.14 .

Proposition 3.11. Soient $(p, k) \in \mathcal{O}$ et $n \geq 1$. Soit $\left.I(p, k)=] H(p, s(p, 1), \ldots, s(p, k-1)), \frac{1}{p^{\sim}}\right]$.

Si $x$ est pris au hasard dans $I(p, k)$ suivant la loi uniforme, la probabilité pour que la suite formée des $k+n$ premiers nombres de son développement soit de résultant $r(p, k)$ est égale $\grave{a}{ }^{n} Z_{p, k}^{p, k}$.

Preuve: D'après le théorème 2.13, l'ensemble des $x \in$ $I(p, k)$ vérifiant la propriété de l'énoncé est la réunion disjointe des ensembles $J(S)$ où $S$ parcourt l'ensemble des $D$-suite de longueur $n+k$, de résultants $r(p, k)$ et commençant par $(p, s(p, 1), \ldots, s(p, k-1))$. Il résulte de la proposition 2.14 que la mesure de Lebesgue de cet ensemble est égale à

$$
\begin{gathered}
r(p, k) \prod_{i=0}^{k-1} \frac{1}{s(p, i)} \sum\left\{\prod_{j=1}^{n} \frac{1}{p_{j}} ;\left(p_{1}, p_{2}, \ldots, p_{n}\right)\right. \\
\text { est }[(p, k),(p, k)] \text {-compatible }\} .
\end{gathered}
$$

Mais d'après les propositions 2.14 et 3.9 , ce dernier nombre est égal au produit de la longueur de $I(p, k)$ par ${ }^{n} Z_{p, k}^{p, k}$.

Les calculs par Maple suivant ce procédé de ${ }^{n} Z_{5,1}^{5,1}$ pour $n=100,200$ et 300 font apparaître des valeurs comprises entre 0,145 et 0,148 .
D'autre part le calcul approché de la densité stationnaire

$$
g=\sum_{(p, i) \in \mathcal{O}} \frac{\alpha_{p, i}}{r(p, i)} e_{p, i}
$$

par la deuxième méthode du paragraphe suivant donne $\alpha_{5,1}=0,145 \ldots$ Tous ces éléments nous amènent à énoncer la conjecture suivante:

Conjecture 3.12. La chaîne $\mathcal{P}$ est ergodique.

\subsection{Conséquences de l'hypothèse de l'ergodicité de la chaîne $\mathcal{P}$}

Dans ce paragraphe nous admettons l'hypothèse de l'ergodicité de la chaîne $\mathcal{P}$. Soit

$$
g=\sum_{(p, k) \in \mathcal{O}} \frac{\alpha_{p, k}}{r(p, k)} e_{p, k}
$$

la densité de probabilité stationnaire de $T$ (proposition 3.4). La transformation $\Phi$ préserve donc la mesure $\mu=$ $g \lambda$. Nous nous intéressons aux propriétés asymptotiques du couple $(\Phi, \mu)$.

Lemme 3.13. Soit $A \subset I$ tel que $\Phi^{-1}(A)=A$. Soit $x \in I$ et soit $S=\left(p_{k}\right)_{0 \leq k \leq n-1}$ le développement d'ordre $n$ de x. Alors

$$
\left.\left.A \cap] H(S), x]=H(S)+\left(\prod_{k=0}^{n-1} \frac{1}{p_{k}}\right) \quad(A \cap] 0, \Phi^{n}(x)\right]\right) .
$$

Preuve: L'égalité précédente avec $n=1$ s'écrit

$$
\left.\left.\left.A \cap] \frac{1}{p_{0}}, x\right]=\frac{1}{p_{0}}+\frac{1}{p_{0}}(A \cap] 0, \Phi(x)\right]\right) .
$$

Afin de la prouver, remarquons que par hypothèse la restriction de $\Phi$ à $\left.] \frac{1}{p_{0}}, x\right]$ concide avec la fonction $t \longrightarrow p_{0} t-1$. Donc

$$
\left.\left.\left.\left.\Phi^{-1}(A) \cap\right] \frac{1}{p_{0}}, x\right]=\left(\frac{1}{p_{0}}+\frac{1}{p_{0}} A\right) \cap\right] \frac{1}{p_{0}}, x\right] .
$$

Comme $\left.\left.\left.] \frac{1}{p_{0}}, x\right]=\frac{1}{p_{0}}+\frac{1}{p_{0}}\right] 0, \Phi(x)\right]$ et que $\Phi^{-1}(A)=A$, l'égalité est vraie pour $n=1$. La démonstration se poursuit en effectuant une récurrence sur $n$.

Lemme 3.14. Soit $A \subset I$ tel que $\Phi^{-1}(A)=$ A. Alors pour toute $D$-suite finie $S=\left(p_{k}\right)_{0 \leq k<n}$, on a:

$$
\left.\left.\left.A \cap J(S)=H(S)+\left(\prod_{k=0}^{n-1} \frac{1}{p_{k}}\right)(A \cap] 0, \operatorname{res}(S)\right]\right) .\right)
$$


Preuve: La relation est vraie si $n=1$. On a en effet:

$$
\left.\left.S=\left(p_{0}\right), \quad J(S)=\right] \frac{1}{p_{0}}, \frac{1}{p_{0}^{\tau}}\right]
$$

et

$$
\begin{aligned}
\Phi^{-1}(A) \cap J(S) & \left.=] \frac{1}{p_{0}}, \frac{1}{p_{0}^{\sim}}\right] \cap\left(\frac{1}{p_{0}}+\frac{1}{p_{0}} A\right) \\
& \left.\left.=\frac{1}{p_{0}}+\frac{1}{p_{0}}(A \cap] 0, r\left(p_{0}, 1\right)\right]\right) .
\end{aligned}
$$

Supposons le lemme établi pour $n \geq 1$. Soit $S=$ $\left(p_{k}\right)_{0 \leq k<n+1}$ une $D$-suite de longueur $n+1$. Puisque

$$
\left.J(S) \subset] \frac{1}{p_{0}}, \frac{1}{p_{0}^{\sim}}\right]
$$

on a

$$
\Phi^{-1}(A) \cap J(S)=\left(\frac{1}{p_{0}}+\frac{1}{p_{0}} A\right) \cap J(S) .
$$

Si $S$ est le développement d'ordre $n+1$ de $\frac{1}{p_{0}}$ l'égalité (3-3) résulte du lemme 3.13. Autrement $S$ possède au moins deux indices de saut. Alors d'après la proposition 2.10 le plus grand indice de saut $k_{m-1}$ de la $D$-suite $S^{\prime}=\left(p_{k}\right)_{1 \leq k<n+1}$ est le même que celui de $S$; par suite d'après le théorème 2.13 ,

$$
J(S)=\frac{1}{p_{0}}+\frac{1}{p_{0}} J\left(S^{\prime}\right),
$$

d'où

$$
\Phi^{-1}(A) \cap J(S)=\frac{1}{p_{0}}+\frac{1}{p_{0}}\left(A \cap J\left(S^{\prime}\right)\right) .
$$

Par l'hypothèse de récurrence,

$$
\left.\left.A \cap J\left(S^{\prime}\right)=H\left(S^{\prime}\right)+\left(\prod_{k=1}^{n} \frac{1}{p_{k}}\right)(A \cap] 0, \operatorname{res}\left(S^{\prime}\right)\right]\right) .
$$

Comme $\operatorname{res}(S)=\operatorname{res}\left(S^{\prime}\right)$ et que $A=\Phi^{-1}(A)$, on en déduit que (3-3) est vraie pour $n+1$. Le lemme est donc établi par récurrence.

Proposition 3.15. En supposant l'ergodicité de la chaîne $\mathcal{P}$, la transformation $\Phi$ est ergodique.

Preuve: Soit $A$ borélien de $I$ tel que $\Phi^{-1}(A)=A$. D'après la proposition 2.14 et le lemme 3.14 , on a pour toute $D$-suite finie $S$ :

$$
\frac{\lambda(A \cap J(S))}{\lambda(J(S))}=\frac{\lambda(A \cap] 0, \operatorname{res}(S)])}{\operatorname{res}(S)} .
$$

Par ailleurs d'après un théorème de Lebesgue, on a pour $\lambda$-presque tout $x \in I$ :

$$
\lim _{\substack{y \rightarrow x, y<x \\ z \rightarrow x \rightarrow x<z}} \frac{\lambda(A \cap[y, z])}{z-y}=\mathbb{1}_{A}(x) .
$$

Il en résulte que si pour $n \geq 0$ et $x \in I, S_{n}(x)$ désigne le développement d'ordre $n$ de $x$, on a pour $\lambda$-presque tout $x \in I$ :

$$
\lim _{n} \frac{\lambda\left(A \cap J\left(S_{n}(x)\right)\right)}{\lambda\left(J\left(S_{n}(x)\right)\right)}=\mathbb{1}_{A}(x) .
$$

Montrons que pour $\lambda$-presque tout $x, \operatorname{res}\left(S_{n}(x)\right)=$ $\frac{2}{3}$ pour une infinité de $n$. La mesure $\mu=g \lambda$ qui est invariante par $\Phi$, est équivalente à $\lambda$. Il découle du théorème de l'éternel retour de Poincaré que pour $\lambda$ presque tout $x \in I$ la trajectoire $\left[\Phi^{n}(x)\right]_{n \geq 0}$ issue de $x$ visite une infinité de fois l'intervalle

$$
] \frac{1}{3}+\frac{1}{3.5}, \frac{1}{3}+\frac{1}{3.3}\right]
$$

cela entraîne que dans le développement de $x$, la séquence $(3,5)$ apparaît une infinité de fois ; comme $5>s(3,1)$ cela prouve notre assertion. Par suite d'après $(3-4)$ et $(3-5), \mathbb{1}_{A}(x)$ est $\lambda$-presque partout égale à une constante, c'est-à-dire que $A=I$ ou $A=\phi \quad \lambda$-pp. Ce qui démontre l'ergodicité du couple $(\Phi, \mu)$.

Le reste de ce sous-paragraphe vient en complément d'une première version de notre travail. Nous améliorons le résultat précédent en montrant que sous l'hypothèse de l'ergodicité de la chaîne $\mathcal{P}$ le couple $(\Phi, \mu)$ est exact. Pour cela nous commençons par établir une formule permettant d'obtenir une expression simple de la puissance nième de l'opérateur de Perron-Frobenius $T$; ce qui permet d'utiliser les résultats de [Lasota and Mackey 94] dans le but de démontrer le caractère statistiquement stable de la transformation $\Phi$.

Rappelons que pour une suite de nombres $S=$ $\left(p_{k}\right)_{0 \leq k \leq n-1}$, nous notons

$$
H(S)=\sum_{\ell=0}^{n-1} \prod_{k=0}^{\ell} \frac{1}{p_{k}} .
$$

Nous écrivons aussi

$$
\Pi(S)=\prod_{k=0}^{n-1} \frac{1}{p_{k}} .
$$

Pour les définitions de $\operatorname{res}(S)$ et de $J(S)$ lorsque $S$ est une $D$-suite nous renvoyons au début du $\S 2.2$. Nous notons $\mathcal{D}_{n}$ l'ensemble des $D$-suites de longueur $n$. 
Proposition 3.16. Soient $f \in L^{1}(I, \lambda)$ et $n \geq 1$. On a pour presque tout $t \in I$ :

$$
T^{n} f(t)=\sum_{S \in \mathcal{D}_{n}} \Pi(S) f(H(S)+t \Pi(S)) \mathbb{1}_{j 0, r e s(S)]}(t) .
$$

Preuve: La relation (3-6) est vraie pour $n=1$, car c'est la formule classique donnant l'expression de $T$ (formule (3-1) au début de ce paragraphe). En effet le résultant d'une $D$-suite de longueur 1 à savoir $(p)$ est égal à $r(p, 1)$. Appliquons l'opérateur $T$ aux deux membres de la relation (3-6) supposée vraie pour $n \geq 1$. On obtient:

$$
\begin{aligned}
T^{n+1} f(t) & =\sum_{S \in \mathcal{D}_{n}} \sum_{p} \Pi([S, p]) f(H([S, p]) \\
& \left.+t \Pi([S, p])) \mathbb{1}_{0, \max }(0, \min (\operatorname{res}(S) p-1, r(p, 1)))\right]^{(t),}
\end{aligned}
$$

où $[S, p]$ désigne la suite $S$ augmentée de l'élément $p$. On a tenu compte du fait que

$$
\begin{aligned}
\mathbb{1}_{0, \text { res }(S)]}\left(\frac{1+t}{p}\right) \mathbb{1}_{0, r(p, 1)]}(t)= \\
\mathbb{1}_{0, \max (0, \min (\operatorname{res}(S) p-1, r(p, 1)))]}(t) .
\end{aligned}
$$

La fonction indicatrice ci-dessus que l'on note $g_{[S, p]}$ est non identiquement nulle si et seulement si $\frac{1}{p}<\operatorname{res}(S)$, ce qui revient au même de dire que la suite $[S, p]$ est une $D$-suite (définition 2.7). Supposons qu'il en soit ainsi. Deux cas se présentent alors:

(1) $\frac{1}{p}<\operatorname{res}(S) \leq \frac{1}{p^{\sim}}$ : on a

$$
\operatorname{res}([S, p])=\operatorname{res}(S) p-1 \leq r(p, 1)
$$

car le plus grand indice de saut de $[S, p]$ est le même que celui de $S$ (définition 2.6); d'où $g_{[S, p]}=$ $\mathbb{1}_{0, \operatorname{res}([S, p])]}$.

(2) $\frac{1}{p^{\sim}}<\operatorname{res}(S)$ : on a

$$
\operatorname{res}([S, p])=r(p, 1)<\operatorname{res}(P) p-1
$$

car le plus grand indice de saut de $[S, p]$ est son dernier indice. Par conséquent on a encore $g_{[S, p]}=$ $\mathbb{1}_{0, \operatorname{res}([S, p])]}$.

Ainsi la relation (3-7) n'est autre que la relation (3-6) où $n$ est remplacé par $n+1$.
Remarque 3.17. La proposition précédente permet une démonstration plus directe de la proposition 3.9.

Nous notons $D(I)$ l'ensemble des densités de probabilité sur $I$, la mesure de référence étant $\lambda$. Pour tout $f \in D(I)$ et tout $n \geq 1$ nous définissons $U_{n} f \in D(I)$ par:

$$
U_{n} f=\sum_{S \in \mathcal{D}_{n}}\left(\int_{J(S)} f(u) d u\right) \frac{1}{\operatorname{res}(S)} \mathbb{1}_{0, r e s(S)]} .
$$

Proposition 3.18. Pour tout $f \in D(I), \lim _{n} \| T^{n} f-$ $U_{n} f \|_{1}=0$.

Preuve: Soit $f \in D(I)$ et $n \geq 1$. D'après la proposition 3.16 et par définition de $U_{n} f$ on a:

$$
\begin{aligned}
&\left\|T^{n} f-U_{n} f\right\|_{1} \leq \sum_{S \in \mathcal{D}_{n}} \int_{0}^{r e s(S)} \mid \Pi(S) f(H(S)+t \Pi(S)) \\
&-\frac{1}{\operatorname{res}(S)} \int_{J(S)} f(u) d u \mid d t .
\end{aligned}
$$

En effectuant le changement de variable $v=H(S)+$ $t \Pi(S)$ dans les intégrales de la somme du second membre ci-dessus, tenant compte du fait que $J(S)=$ ] $H(S), H(S)+\operatorname{res}(S) \Pi(S)$ ] (théorème 2.13), on obtient:

$\left\|T^{n} f-U_{n} f\right\|_{1} \leq \sum_{S \in \mathcal{D}_{n}} \int_{J(S)}\left|f(v)-\frac{1}{\operatorname{res}(S)} \int_{J(S)} f(u) d u\right| d v$.

On reconnait au second membre: $\left\|f-E\left(f / \mathcal{B}_{n}\right)\right\|_{1}$ où $\mathcal{B}_{n}$ désigne la tribu finie engendrée par les $J(S), S$ parcourant $\mathcal{D}_{n} ; E\left(f / \mathcal{B}_{n}\right)$ étant l'espérance conditionnelle de $f$ par rapport à $\mathcal{B}_{n}$. Du fait même de l'existence de la correspondande bijective entre $I$ et l'ensemble des $D$-suites, la tribu engendrée par $\bigcup_{n} \mathcal{B}_{n}$ est la tribu de Borel de $I$. Or par un théorème classique on a $\lim _{n}\left\|f-E\left(f / \mathcal{B}_{n}\right)\right\|_{1}=$ 0 pour tout $f \in L^{1}(I, \lambda)$.

Pour les définitions de l'ensemble $\mathcal{O}$, de l'espace $\mathcal{E}$ et de la matrice $\mathcal{Z}$ nous renvoyons au début de ce paragraphe. Soit $F$ l'application de $l^{1}(\mathcal{O})$ dans $L^{1}(I, \lambda)$ définie par: $\left(\alpha_{p, k}\right)_{(p, k) \in \mathcal{O}} \longrightarrow \sum \frac{\alpha_{p, k}}{r(p, k)} e_{p, k}$ (on rappelle que $e_{p, k}$ désigne $\left.\mathbb{1}_{0, r(p, k)]}\right)$. L'image de $F$ est l'espace $\mathcal{E}$. Nous avons vu que cet espace est stable par l'opérateur de Perron-Frobenius $T$ associé à $\Phi$. Soit $T_{\mid \mathcal{E}}$ la réduction de $T$ à $\mathcal{E}$. La matrice de $T_{\mid \mathcal{E}}$ dans la base de Schauder normalisée $\left(e_{p, k} / r(p, k)\right)_{(p, k) \in \mathcal{O}}$ n'est autre que $\mathcal{Z}$. Soit $K=F^{-1} T_{\mid \mathcal{E}} F$ l'opérateur de $l^{1}(\mathcal{O})$ dans $l^{1}(\mathcal{O})$ transmué de $T_{\mid \mathcal{E}}$ par l'application $F$. La matrice de $K$ dans la base canonique étant aussi $\mathcal{Z}$ on voit que $K$ est Markovien. 
Nous notons $D(\mathcal{O})$ l'ensemble des densités sur $\mathcal{O}$, la mesure de référence étant bien entendu la mesure $\nu$ telle que $\nu(\{c\})=1$ pour tout $c \in \mathcal{O}$. D'après l'hypothèse d'ergodicité de la chaîne de Markov $\mathcal{P}$ Il existe une densité stationnaire unique $\alpha$ pour $K$. De plus pour toute autre densité $\beta$ de $D(\mathcal{O})$ on a $K^{n} \beta \longrightarrow \alpha$ simplement sur $\mathcal{O}$ (voir le début du $§ 3.1$ ).

On voit facilement que $K$ est constrictif [Lasota and Mackey 94, definition 5.3.2]. En effet soit $B$ un sous ensemle fini de $\mathcal{O}$ tel que $\sum_{(p, k) \in B} \alpha_{p, k}>1 / 2$. Pour toute densité $\beta$ de $D(\mathcal{O})$ il existe un entier $n_{0}(\beta)$ tel que pour tout $n \geq n_{0}(\beta), \sum_{(p, k) \in B}\left(K^{n} \beta\right)_{p, k}>1 / 2$. En choisissant $\delta$ quelconque avec $0<\delta<1$, on a pour tout $E \subset \mathcal{O}$ tel que $\nu(E)<\delta$ et tout $n \geq n_{0}(\beta)$, $\sum_{(p, k) \in(\mathcal{O} / B) \cup E}\left(K^{n} \beta\right)_{p, k}<1 / 2$; ce qui montre bien la constrictivité de $K$.

On peut alors appliquer à $K$ le théorème 5.6 .1 de [Lasota and Mackey 94]; on voit ainsi que la suite $\left\{K^{n}\right\}$ est asymptotiquement stable [Lasota and Mackey 94 , definition 5.6.1], à savoir que pour tout $\beta \in D(\mathcal{O}), K^{n} \beta \longrightarrow \alpha$ dans $l^{1}(\mathcal{O})$.

Soit $g=\sum_{(p, k) \in \mathcal{O}} \frac{\alpha_{p, k}}{r(p, k)} e_{p, k}$ la densité stationnaire de $T$ (proposition 3.4). Il résulte de ce qui précède que pour tout $f \in D(I) \cap \mathcal{E}, T^{n} f \longrightarrow g$ dans $L^{1}(I, \lambda)$. Le théorème suivant affirme qu'il en est de même pour tout $f \in D(I)$; ce qui veut dire que $\Phi$ est statistiquement stable [Lasota and Mackey 94, definition 5.6.2].

Théorème 3.19. Sous l'hypothèse de l'ergodicité de la chaîne de Markov $\mathcal{P}$, la transformation $\Phi$ est statistiquement stable.

Preuve: Soient $f \in D(I)$ et $\epsilon>0$. D'après la proposition 3.18 il existe un entier $n$ et $f^{*} \in D(I) \cap \mathcal{E}$ tels que $\| T^{n} f-$ $f^{*} \|_{1}<\epsilon / 2$. D'après la discussion précédente il existe $k$ tel que $\left\|T^{k} f^{*}-g\right\|_{1}<\epsilon / 2$. D'où $\left\|T^{n+k} f-g\right\|_{1}<\epsilon$; on en déduit que pour tout $f \in D(I), T^{n} f \longrightarrow g$ dans $L^{1}(I, \lambda)$.

D'après [Lasota and Mackey 94, proposition 5.6.2] on peut alors énoncer le résultat suivant:

Corollaire 3.20. Sous l'hypothèse d'ergodicité de la chaîne de Markov $\mathcal{P}$, le couple $(\Phi, \mu)$ où $\mu=g \lambda$ est exact et en particulier mélangeant.

\subsection{Relations vérifiées par une distribution invariante de $\mathcal{P}$}

Dans ce paragraphe nous déterminons les rapports $\frac{\alpha_{p, k}}{\alpha_{p, 1}}$ pour $(p, k) \in \mathcal{O}$ en supposant que $\alpha$ est une distribution invariante de $\mathcal{P}$. Ces relations sont utilisées au $\oint 5$ dans les algorithmes de calcul de la densité stationnaire $g$.

Rappelons que d'après la relation (3-2) du début de ce paragraphe on a pour tout $(p, k) \in \mathcal{O}$ :

$T\left(e_{p, k}\right)=\left\{\begin{array}{l}\frac{1}{s(p, k)} e_{p, k+1}+\sum_{p^{\prime}>s(p, k)} \frac{1}{p^{\prime}} e_{p^{\prime}, 1} \text { si } k<h(p)-1, \\ \frac{1}{s(p, k)} e_{p, j(p)}+\sum_{p^{\prime}>s(p, k)} \frac{1}{p^{\prime}} e_{p^{\prime}, 1} \text { si } k=h(p)-1 .\end{array}\right.$

Reportons cette expression dans la somme $\sum_{(p, k) \in \mathcal{O}} \frac{\alpha_{p, k}}{r(p, k)} T\left(e_{p, k}\right)$ et après regroupement des termes semblables, identifions le résultat à $g=\sum \frac{\alpha_{p, k}}{r(p, k)} e_{p, k}$ (voir lemme 3.1).

On voit alors que pour tout $p>5$ et tout $k, 2 \leq k \leq$ $h(p)-1$ :

$\frac{\alpha_{p, k}}{r(p, k)}= \begin{cases}\frac{\alpha_{p, k-1}}{s(p, k-1) r(p, k-1)} & \text { si } k \neq j(p), \\ \frac{\alpha_{p, j(p)-1}}{r(p, j(p)-1) s(p, j(p)-1)} & \\ +\frac{\alpha_{p, h(p)-1}}{r(p, h(p)-1) s(p, h(p)-1)} & \text { si } k=j(p) \text { et } j(p) \geq 2 .\end{cases}$

(D'ailleurs il apparait aussi que $4 \alpha_{2,1}=\alpha_{5,1}$ ).

On en déduit les relations suivantes:

$$
\forall \ell, \quad 2 \leq \ell<j(p), \quad \frac{\alpha_{p, \ell}}{\alpha_{p, 1}}=\frac{r(p, \ell)}{r(p, 1)} \prod_{k=1}^{\ell-1} \frac{1}{s(p, k)},
$$

$\forall \ell, j(p)<\ell<h(p), \quad \frac{\alpha_{p, \ell}}{\alpha_{p, j(p)}}=\frac{r(p, \ell)}{r(p, j(p))} \prod_{k=j(p)}^{\ell-1} \frac{1}{s(p, k)}$,

et si $j(p) \geq 2$,

$$
\frac{\alpha_{p, j(p)}}{\alpha_{p, 1}}=\frac{r(p, j(p))}{r(p, 1)} \prod_{k=1}^{j(p)-1} \frac{1}{s(p, k)}\left(1-\prod_{k=j(p)}^{h(p)-1} \frac{1}{s(p, k)}\right)^{-1} .
$$

\section{CALCUL APPROCHÉ DE LA DENSITÉ STATIONNAIRE}

L'hypothèse de l'ergodicité de $\mathcal{P}$ étant admise, le but de cette partie est la détermination d'une approximation la plus fine possible de la densité stationnaire $g=$ $\sum \alpha_{p, k} / r(p, k) \mathbb{1}_{0, r(p, k)]}$. Les deux méthodes exposées cidessous sont complémentaires et sont fondées sur une démarche heuristique qui utilise la méthode de [Ulam 60] d'approximation matricielle de l'opérateur $T$ tout en 
conservant le terme reste sous la forme d'un opérateur intégral.

Rappelons la formule (3-1) du paragraphe 3 :

$$
T f(t)=\sum_{n=1}^{\infty} \frac{1}{p_{n}} f\left(\frac{1+t}{p_{n}}\right) \mathbb{1}_{\left[0, p_{n} / p_{n}^{\sim}-1\right.}(t),
$$

où $p_{n}$ désigne le nombre premier de rang $n$.

Un entier $\mathrm{N}$ étant fixé, nous définissons l'opérateur tronqué $T_{N}$ par la somme partielle d'indice $\mathrm{N}$ de la série ci-dessus et nous notons $\widetilde{T}_{N}$ l'opérateur reste $T-T_{N}$.

Une première étape consiste à remplacer la série définissant $\widetilde{T}_{N} f(t)$ par une intégrale. Pour cela nous utilisons la fonction $G$ sur $\mathbb{R}_{+}$définie par (log désignant toujours par la suite le logarithme népérien):

$$
\begin{aligned}
G(x)=(x+2,1362) & {[\log (x+2,1362)} \\
+ & \left.\log \log (x+2,1362)-\frac{21}{22}\right],
\end{aligned}
$$

fonction qui présente l'intérêt de fournir un lissage correct de la suite $n \longrightarrow p_{n}$. Elle s'obtient en partant de l'encadrement [Ellison et Mendès France 75, p. 25]: $\forall n \geq 21$,

$n\left(\log n+\log \log n-\frac{3}{2}\right)<p_{n}<n\left(\log n+\log \log n-\frac{1}{2}\right)$

et en procédant à l'aide de Maple à un ajustement empirique de son graphe avec celui de la suite $n \longrightarrow p_{n}$ pour $n \leq 30000$.

D'autre part grâce à la formule ([Ellison et Mendès France 75], p. 32) (où $a=0,261 \ldots$ ):

$$
\sum_{p<x} \frac{1}{p}=a+\log \log (x)+O\left(\frac{1}{\log x}\right)
$$

on a pour $x$ assez grand:

$$
\sum_{1 \leq n \leq x} \frac{1}{p_{n}} \simeq a+\log \log (G(x)) .
$$

En fait nous n'utilisons que les premiers termes du développement asymptotique du deuxième membre, à savoir la fonction

$$
\begin{aligned}
L(x)=a+ & \log \log (x) \\
& +\frac{\log \log (x)}{\log (x)}+\frac{\log \log (x)-\frac{3}{4}-\frac{1}{2} \log \log (x)^{2}}{\log (x)^{2}} .
\end{aligned}
$$

L'autre intérêt de $L$ est de fournir une bonne approximation d'une primitive de $\frac{1}{G(x)}$ pour $x$ grand.

\subsection{Description de la première méthode d'approx- imation. Conjecture concernant la partie princi- pale de la densité stationnaire au voisinage de $\mathbf{0}$.}

Cette méthode est fondée sur le raisonnement heuristique suivant: d'après le théorème des nombres premiers (voir [Ellison et Mendès France 75]) $p_{n}-p_{n-1}$ est "en moyenne" égal à $\log n$ et comme $p_{n} \sim n \log n$ on voit que $\frac{p_{n}}{p_{n}^{\sim}}-1$ se comporte "en moyenne" comme $\frac{1}{n}$. Cela nous amène pour la première méthode à utiliser la forme simplifiée $\widetilde{T}_{N}^{(1)}$ de l'opérateur $\widetilde{T}_{N}$ définie par:

$$
\widetilde{T}_{N}^{(1)} f(t)=\mathbb{1}_{\left.0, \frac{1}{N}\right]}(t) \sum_{N \leq n<\frac{1}{t}} \frac{1}{p_{n}} f\left(\frac{1+t}{p_{n}}\right),
$$

ou encore sous forme intégrale:

$$
\widetilde{T}_{N}^{(1)} f(t)=\mathbb{1}_{\left.0, \frac{1}{N}\right]}(t) \int_{N}^{1 / t} f\left(\frac{1+t}{G(x)}\right) \frac{d x}{G(x)} ;
$$

ce qui donne, en faisant $t=0$ sous l'intégrale puis en effectuant le changement de variable $u=L(x)$ (sachant que $\left.d u=\frac{d x}{G(x)}\right)$ :

$$
\widetilde{T}_{N}^{(1)} f(t)=\mathbb{1}_{\left.0, \frac{1}{N}\right]}(t) \int_{L(N)}^{L(1 / t)} f\left(\frac{1}{G\left(L^{-1}(u)\right)}\right) d u .
$$

Remarque 4.1. Dans le cas particulier où $f=\mathbb{1}_{0, s]}$ avec $P(s)<p_{N+1}$ on justifie directement l'approximation

$$
\widetilde{T}_{N}^{(1)} f(t) \simeq\left[L\left(\frac{1}{t}\right)-L(N)\right] \mathbb{1}_{\left.0, \frac{1}{N}\right]}(t)
$$

en s'appuyant sur le fait que dans ce cas on a d'après la formule (3-2) du début du $\S 3$ :

$$
\left.\widetilde{T}_{N} f(t)=\sum_{n \geq N+1} \frac{1}{p_{n}} \mathbb{1}_{0, p_{n} / p_{n}^{\sim}-1}\right]^{(t) .}
$$

Cela s'applique en particulier au cas où $f=\mathbb{1}_{0, r(p, k)]}$ avec $p \leq p_{N}$ et $1 \leq k \leq h(p)-1$.

En effet du fait que $\frac{1}{p^{\sim}} \leq r(p, k)$, on a $P(r(p, k)) \leq$ $p<p_{N+1}$.

Pour la définition de la fonction $h$, ainsi que celles de $\mathcal{E}$, de $\mathcal{O}$, de $e_{p, k}$ et de $\mathcal{A}$ nous renvoyons au début du $\S 3$. D'après $(4-1)$ et la remarque précédente un vecteur propre de $T_{N}+\widetilde{T}_{N}^{(1)}$ est de la forme

$$
f(t)=\sum_{(p, k) \in \mathcal{O}, p \leq p_{N}} \alpha_{p, k} e_{p, k}(t)+\mathbb{1}_{\left.0, \frac{1}{N}\right]}(t) \int_{L(N)}^{L(1 / t)} \varphi(x) d x,
$$

où $\alpha_{p, k} \in \mathbb{R}$ et $\varphi$ est une fonction continue sur $[L(N), \infty[$. 
4.1.1 Notation. Nous écrivons $O_{N}=\{(p, k) \in \mathcal{O} ; p \leq$ $\left.p_{N}\right\}$ et nous notons $E_{N}$ le vecteur ligne dont les composantes sont les fonctions $e_{p, k},(p, k) \in O_{N}$. Ainsi pour tout vecteur colonne $V=\left[\alpha_{p, k}\right]_{(p, k) \in O_{N}}$, la somme $\sum_{(p, k) \in O_{N}} \alpha_{p, k} e_{p, k}(t)$ s'écrit plus simplement $E_{N}(t) V$. D'autre part $\mathcal{E}_{N}$ désigne le sous-espace de $\mathcal{E}$ engendré par les $e_{p, k},(p, k) \in O_{N}$ et $p r_{N}$ est la projection canonique de $\mathcal{E}$ sur $\mathcal{E}_{N}$. Enfin $\mathcal{A}_{N}$ est la matrice de l'endomorphisme $p r_{N} \circ T_{N} \mid \mathcal{E}_{N}$ dans la base $\left[e_{p, k}\right]_{(p, k) \in O_{N}}$; (cette matrice est donc un bloc de la matrice $\mathcal{A}$ ). Dans les propositions 4.1 et 4.4 plus bas $\delta_{p, 1}$ désigne le vecteur colonne élémentaire de $\mathbb{R}^{O_{N}}$ associé à $e_{p, 1},(p \leq N)$. Nous adoptons la notation $x \vee y$ pour $\max (x, y)$.

Soit $\mathcal{L}_{N}^{(1)}$ le sous-espace de $L^{1}(I, \lambda)$ constitué des fonctions $f$ pour lesquelles il existe un couple $(V, \varphi)$ où $V \in \mathbb{R}^{O_{N}}$ et $\varphi$ est une fonction continue, tel que:

$$
f(t)=E_{N}(t) V+\mathbb{1}_{\left.0, \frac{1}{N}\right]}(t) \int_{L(N)}^{L(1 / t)} \varphi(x) d x .
$$

En première approximation $\mathcal{L}_{N}^{(1)}$ est invariant par $T_{N}+\widetilde{T}_{N}^{(1)}$. Plus précisément nous énonçons la proposition suivante:

Proposition 4.2. L'opérateur $T_{N}+\widetilde{T}_{N}^{(1)}$ est asymptotiquement proche de l'endomorphisme noté $\Gamma_{N}^{1}$ de $\mathcal{L}_{N}^{(1)}$ qui à $f \in \mathcal{L}_{N}^{(1)}$ représenté selon (4-3) par le couple $(V, \varphi)$, associe l'élément représenté par $(W, \psi)$ où:

$$
W=\mathcal{A}_{N} V+\sum_{1 \leq n \leq N}\left[\frac{1}{p_{n}} \int_{L(N)}^{L\left(N \vee p_{n}\right)} \varphi(x) d x\right] \delta_{p_{n}, 1}
$$

et

$$
\psi(x)=s(V)+\int_{L(N)}^{x} \varphi(u) d u, \quad x \geq L(N),
$$

$(s(V)$ désignant la somme des composantes de $V)$.

Preuve: Soit $f \in \mathcal{L}_{N}^{(1)} ;$ posons $f_{1}(t)=E_{N}(t) V$ et

$$
f_{2}(t)=\mathbb{1}_{\left.0, \frac{1}{N}\right]}(t) \int_{L(N)}^{L(1 / t)} \varphi(x) d x .
$$

Il résulte de (4-2) (voir la remarque plus haut) que

$$
T f_{1}(t) \simeq E_{N}(t) \mathcal{A}_{N} V+s(V) \mathbb{1}_{\left.0, \frac{1}{N}\right]}(t) \int_{L(N)}^{L(1 / t)} d u .
$$

D'autre part on a pour tout $u \geq L(N): L^{-1}(u) \geq N$, d'où $G\left(L^{-1}(u)\right) \geq G(N) \geq N$ et par suite

$$
f_{2}\left(\frac{1}{G\left(L^{-1}(u)\right)}\right)=\int_{L(N)}^{L\left(G\left(L^{-1}(u)\right)\right)} \varphi(x) d x .
$$

Comme $L\left(G\left(L^{-1}(u)\right)\right)=u+u e^{-u}(1+o(1))$, on a pratiquement $L\left(G\left(L^{-1}(u)\right)\right)=u$. Ainsi:

$$
\widetilde{T}_{N}^{(1)} f_{2}(t) \simeq \mathbb{1}_{]_{0, \frac{1}{N}}}(t) \int_{L(N)}^{L(1 / t)}\left(\int_{L(N)}^{u} \varphi(x) d x\right) d u .
$$

Enfin en simplifiant

$$
T_{N} f_{2}(t) \simeq \sum_{1 \leq n \leq N} \frac{1}{p_{n}} f_{2}\left(\frac{1}{p_{n}}\right) e_{p_{n}, 1}(t),
$$

on voit que $T f_{2}$ est proche de la fonction de $\mathcal{L}_{N}^{(1)}$ représentée selon (4-3) par le couple

$$
\left(\sum_{n=1}^{N} \frac{1}{p_{n}} f_{2}\left(\frac{1}{p_{n}}\right) \delta_{p_{n}, 1}, \quad \int_{L(N)}^{x} \varphi(u) d u\right) .
$$

En ajoutant les deux approximations de $T f_{1}$ et $T f_{2}$ ainsi définies on obtient la fonction $\Gamma_{N}^{1} f$ de $\mathcal{L}_{N}^{(1)}$ représentée par le couple $(W, \psi)$ de l'énoncé.

On obtient une approximation $g_{N}^{(1)}$ de $g$ sous forme du vecteur propre normalisé de $\Gamma_{N}^{1}$ de valeur propre dominante $\mu_{N}$. Le couple $(V, \varphi)$ représentant $g_{N}^{(1)}$ vérifie:

$$
\left\{\begin{array}{l}
s(V)+\int_{L(N)}^{x} \varphi(u) d u=\mu_{N} \varphi(x) \\
\text { et } \mathcal{A}_{N} V+\sum_{1 \leq n \leq N}\left(\frac{1}{p_{n}} \int_{L(N)}^{L\left(N \vee p_{n}\right)} \varphi(x) d x\right) \delta_{p_{n}, 1}=\mu_{N} V .
\end{array}\right.
$$

On en déduit d'une part que pour tout $x \geq L(N)$,

$$
\varphi(x)=\frac{1}{\mu_{N}} s(V) \exp \left(\frac{1}{\mu_{N}}(x-L(N))\right) .
$$

Soit d'autre part $D_{N}\left(\mu_{N}\right)$ la matrice carrée construite sur l'ensemble d'indices $O_{N}$ telle que pour tout $n \leq N$ sa ligne d'indice $\left(p_{n}, 1\right)$ soit formée de

$$
\frac{1}{p_{n}}\left[\exp \left(\frac{1}{\mu_{N}}\left(L\left(N \vee p_{n}\right)-L(N)\right)\right)-1\right]
$$

répété $\operatorname{card}\left(O_{N}\right)$ fois, tandis que toutes ses autres lignes sont nulles.

Compte tenu de (4-5) la relation (4-4) conduit à

$$
\left[\mathcal{A}_{N}+D_{N}\left(\mu_{N}\right)\right] V=\mu_{N} V
$$

La valeur de $\mu_{N}$ maximale pour laquelle l'équation (4-6) admet une solution non nulle est calculée par approximations successives, ce qui détermine simultanément $V$. On obtient alors $\varphi$ à partir de (4-5) et enfin $g_{N}^{(1)}$ par normalisation dans $L^{1}(I, \lambda)$ de la fonction

$t \longrightarrow E_{N}(t) V+\mathbb{1}_{\left.0, \frac{1}{N}\right]}(t) s(V)\left[\exp \left(\frac{1}{\mu_{N}}(L(1 / t)-L(N))\right)-1\right]$. 
Nous trouvons $\mu_{200}=0,997 \ldots$ et $\mu_{600}=0,999 \ldots$. Il y a donc tout lieu de penser que $\mu_{N} \longrightarrow 1$ et puisque $\exp (L(1 / t)) \sim \log (1 / t)$, nous énonçons la conjecture suivante:

Conjecture 4.3. $\quad k=\lim _{t \rightarrow 0} g(t) / \log (1 / t)$ existe et $k \neq 0$.

Avec $N=600$ nous obtenons $k \simeq 0,78$. Une meilleure estimation de cette constante est donnée au prochain paragraphe.

\subsection{Seconde méthode d'approximation fondée sur l'heuristique de H.Cramér}

Afin d'accélérer la convergence de $g_{N}$ vers $g$ nous définissons une approximation plus fine de l'opérateur $T$ en introduisant un terme qui prend en compte les fluctuations erratiques de la suite

$$
n \longrightarrow\left(\frac{p_{n}}{p_{n}^{\sim}}-1\right) .
$$

Pour cela nous utilisons le modèle heuristique de [Cramér 36] dont voici la description. Soit $\left(U_{n}\right)_{n>2}$ un suite d'urnes contenant des boules noires et blanches, la probabilité de tirer une blanche de $U_{n}$ étant $\frac{1}{\log n}$. Considérons l'expérience aléatoire consistant à tirer de manière indépendante une boule dans chaque urne. Soit pour tout entier $n \geq 1$ la variable aléatoire $\mathbb{X}_{n}$ donnant le rang du nième tirage produisant une boule blanche. Selon cette heuristique, la suite $\left(p_{n}\right)_{n \geq 1}$ est vue comme une réalisation du processus $\left(\mathbb{X}_{n}\right)_{n \geq 1}$. A partir de ce modèle H.Cramér [19] a montré que pour tout $c \geq 0$ la probabilité de l'évènement $p_{n+1}-p_{n}>c \log p_{n}^{2}$ est équivalente à $A p_{n}^{-c}$, (on a $A \simeq 1-0,4 c$ expérimentalement); de plus on a presque sûrement $\mathbb{X}_{n+1}-\mathbb{X}_{n} \leq \log \mathbb{X}_{n}^{2}$ pour tout $n$.

Par conséquent l'inégalité suivante est pratiquement toujours vérifiée:

$$
\frac{2}{p_{n}^{\sim}} \leq \frac{p_{n}}{p_{n}^{\sim}}-1 \leq \frac{\log p_{n}^{2}}{p_{n}} .
$$

Nous posons pour $x>1$ :

$$
\mu(x)=\frac{2}{G(x)} \quad \text { et } \quad \nu(x)=\frac{\log G(x)^{2}}{G(x)},
$$

et plus bas $\mu^{-1}, \nu^{-1}$ désignent les fonctions inverses de $\mu$ et de $\nu$ sur I.

Nous notons pour $t \in I$ et $x>1$ :

$$
\begin{aligned}
\gamma(t, x) & =\frac{t G(x)}{\log G(x)^{2}} \quad \text { et } \\
F(t, x) & =1-G(x)^{-\gamma(t, x)}(1-0,4 \gamma(t, x)) .
\end{aligned}
$$

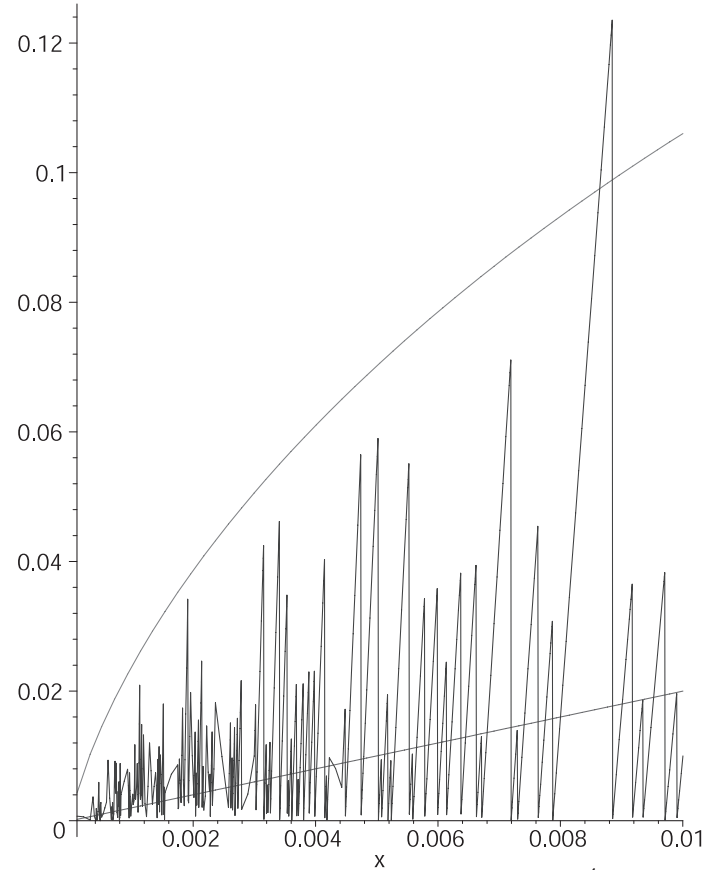

FIGURE 1. Fonction $\Phi$ sur l'intervalle $\left[1 / 10^{4}, 1 / 50\right]$ avec les fonctions $f_{1}: x \rightarrow 2 x$ et $f_{2}: x \rightarrow 1 / 2 x \log (x)^{2}$; $\left(f_{1}(x)\right.$ et $f_{2}(x)$ sont les parties principales de $\mu(n)$ et $1 / 2 \nu(n)$, si $n$ est l'entier tel que $\left.p_{n}=P(x)\right)$.

Rappelons enfin que $\frac{p_{n}}{p_{n}^{\sim}}-1$ s'écrit aussi $r\left(p_{n}, 1\right)$.

D'après la discussion précédente, pour $t \in I$ et pour $\mathrm{n}$ assez grand, la probabilité de l'événement $t \leq r\left(p_{n}, 1\right)$ est égale à $p_{n}^{-\gamma(t, n)}(1-0,4 \gamma(t, n))$. Par conséquent, pour $N \in \mathbb{N}$ et $t \in I$ fixés, l'espérance de $\widetilde{T}_{N} f(t)$ pour $f \in$ $L^{1}(I, \lambda)$ est donnée par

$$
\begin{aligned}
& \sum_{N<n}^{n \leq N \vee \mu^{-1}(t)} \frac{1}{p_{n}} f\left(\frac{1+t}{p_{n}}\right) \\
& \quad+\sum_{N \vee \mu^{-1}(t)<n}^{n \leq N \nu^{-1}(t)} f\left(\frac{1+t}{p_{n}}\right) p_{n}^{-(1+\gamma(t, n))}(1-0,4 \gamma(t, n)) .
\end{aligned}
$$

Nous définissons l'opérateur $\widetilde{T}_{N}^{(2)}$ en posant $\widetilde{T}_{N}^{(2)} f(t)$ égale à l'expression ci-dessus. Puis comme au paragraphe précédent nous remplaçons les sommes $\sum_{n \geq N}$ par des intégrales en y effectuant la même simplification $(t=0$ sous l'intégrale); cela donne après changement de variable:

$$
\begin{aligned}
\widetilde{T}_{N}^{(2)} f(t)= & \int_{L(N)}^{L\left(N \vee \nu^{-1}(t)\right)} f\left(\frac{1}{G\left(L^{-1}(u)\right)}\right) d u- \\
& \int_{L\left(N \vee \nu^{-1}(t)\right)}^{L\left(N \vee \nu^{-1}(t)\right)} f\left(\frac{1}{G\left(L^{-1}(u)\right)}\right) F\left(t, L^{-1}(u)\right) d u .
\end{aligned}
$$


Posons

$$
R_{N}(f, t)=\frac{\int_{L\left(N \vee \mu^{-1}(t)\right)}^{L\left(N \vee \nu^{-1}(t)\right)} f\left(\frac{1}{G\left(L^{-1}(u)\right)}\right) F\left(t, L^{-1}(u)\right) d u}{\int_{L\left(N \vee \mu^{-1}(t)\right)}^{L\left(N \vee \nu^{-1}(t)\right)} f\left(\frac{1}{G\left(L^{-1}(u)\right)}\right) d u .}
$$

Du fait que selon la conjecture précédente $g$ est asymptotiquement proportionnelle à - log et que d'autre part $R_{N}(.,$.$) est homogène de degré 0$ par rapport à son premier argument, on a $R_{N}(g, t) \simeq R_{N}(-\log , t)$. De plus $\log \left(G\left(L^{-1}(u)\right)\right)$ est pratiquement égal à $e^{u}$ (voir paragraphe précédent). Par suite on a:

$R_{N}(-\log , t) \simeq \frac{\int_{L\left(N \vee \mu^{-1}(t)\right)}^{L\left(N \vee \nu^{-1}(t)\right)} e^{u} F\left(t, L^{-1}(u)\right) d u}{\left(\exp \left(L\left(N \vee \nu^{-1}(t)\right)\right)-\exp \left(L\left(N \vee \mu^{-1}(t)\right)\right)\right.}$.

Pour simplifier l'écriture nous notons par la suite

$$
\theta_{N}(t)=R_{N}(-\log , t)
$$

Soit $T_{N}^{*}$ l'opérateur positif sur $L^{1}(I, \lambda)$ défini par

$$
\begin{aligned}
T_{N}^{*} f(t)= & T_{N} f(t)+\int_{L(N)}^{L\left(N \vee \nu^{-1}(t)\right)} f\left(\frac{1}{G\left(L^{-1}(u)\right)}\right) d u \\
& -\theta_{N}(t) \int_{L\left(N \vee \mu^{-1}(t)\right)}^{L\left(N \vee \nu^{-1}(t)\right)} f\left(\frac{1}{G\left(L^{-1}(u)\right)}\right) d u .
\end{aligned}
$$

D'après ce qui précède on peut chercher une approximation $g_{N}^{(2)}$ de $g$ comme vecteur propre de $T_{N}^{*}$. Dans ce but nous procédons à une ultime simplification de $T_{N}^{*}$ afin de permettre le calcul de ses éléments propres par un programme sous Maple. Soit $\mathcal{L}_{N}^{(2)}$ le sous-espace de $L^{1}(I, \lambda)$ constitué des fonctions de la forme:

$$
\begin{aligned}
& f(t)=E_{N}(t) V+P\left(L\left(N \vee \nu^{-1}(t)\right)\right)-P(L(N)) \\
& -\theta_{N}(t)\left[P\left(L\left(N \vee \nu^{-1}(t)\right)\right)-P\left(L\left(N \vee \mu^{-1}(t)\right)\right)\right],
\end{aligned}
$$

où $V$ est un vecteur colonne de $\mathbb{R}^{O_{N}}$ et $u \longrightarrow P(u)$ est une fonction continue sur $[L(N), \infty[$.

En première approximation $\mathcal{L}_{N}^{(2)}$ est invariant par $T_{N}^{*}$. Plus précisément nous énonçons la proposition suivante:

Proposition 4.4. L'opérateur $T_{N}^{*}$ est asymptotiquement proche de l'endomorphisme $\Gamma_{N}^{*}$ de $\mathcal{L}_{N}^{(2)}$ qui à $f \in \mathcal{L}_{N}^{(2)}$ représentée selon (4-9) par le couple $(V, P)$ associe l'élément représenté par le couple $(W, Q)$ tel que:

$$
\begin{aligned}
& W=\mathcal{A}_{N} V+\sum_{n=1}^{N} \frac{1}{p_{n}} {\left[P\left(L\left(N \vee \nu^{-1}\left(\frac{1}{p_{n}}\right)\right)\right)-P(L(N))\right.} \\
&-\theta_{N}\left(\frac{1}{p_{n}}\right)\left(P\left(L\left(N \vee \nu^{-1}\left(\frac{1}{p_{n}}\right)\right)\right)\right. \\
&\left.\left.-P\left(L\left(N \vee \mu^{-1}\left(\frac{1}{p_{n}}\right)\right)\right)\right)\right] \delta_{p_{n}, 1},
\end{aligned}
$$

et $Q(u)$ est une primitive de la fonction

$$
\begin{aligned}
u \longrightarrow s(V) & +P(M(u))-P(L(N)) \\
& -\widetilde{\theta}_{N}(u)[P(M(u))-P(m(u))] ;
\end{aligned}
$$

où $s(V)$ désigne la somme des composantes de $V$ et $M, m$ et $\widetilde{\theta}_{N}$ sont les fonctions définies par:

$$
\begin{aligned}
& M(u)=L\left(N \vee \nu^{-1}\left(\frac{1}{G\left(L^{-1}(u)\right)}\right)\right), \\
& m(u)=L\left(N \vee \mu^{-1}\left(\frac{1}{G\left(L^{-1}(u)\right)}\right)\right), \\
& \widetilde{\theta}_{N}(u)=\theta_{N}\left(\frac{1}{G\left(L^{-1}(u)\right)}\right) .
\end{aligned}
$$

(Rappelons que $\delta_{p_{n}, 1}$ désigne le vecteur colonne élémentaire de $\mathbb{R}^{O_{N}}$ associé à $\left.e_{p_{n}, 1}\right)$.

Preuve: Soit $f \in \mathcal{L}_{N}^{(2)}$. Ecrivons $f=f_{1}+f_{2}$ où $f_{1}(t)=$ $E_{N}(t) V$ et

$$
\begin{aligned}
f_{2}(t) & =P\left(L\left(N \vee \nu^{-1}(t)\right)\right)-P(L(N)) \\
& -\theta_{N}(t)\left[P\left(L\left(N \vee \nu^{-1}(t)\right)\right)-P\left(L\left(N \vee \mu^{-1}(t)\right)\right)\right] .
\end{aligned}
$$

On a pour tout $(p, k) \in O_{N}$ :

$$
\frac{1}{G(N)} \leq \frac{1}{p^{\sim}} \leq r(p, k)
$$

et par conséquent pour tout $u \geq L(N)$,

$$
\frac{1}{G\left(L^{-1}(u)\right)} \leq r(p, k) .
$$

Donc

$$
\begin{aligned}
T_{N}^{*}\left(e_{p, k}\right)(t) & =T_{N}\left(e_{p, k}\right)(t)+L\left(N \vee \nu^{-1}(t)\right)-L(N) \\
& -\theta_{N}(t)\left(L\left(N \vee \nu^{-1}(t)\right)-L\left(N \vee \mu^{-1}(t)\right)\right) .
\end{aligned}
$$

On en déduit que $T_{N}^{*} f_{1} \simeq \Gamma_{N}^{*} f_{1}$ où $\Gamma_{N}^{*} f_{1}$ est représentée selon (4-9) $\operatorname{par}\left(\mathcal{A}_{N} V, s(V) \int d u\right)$. 


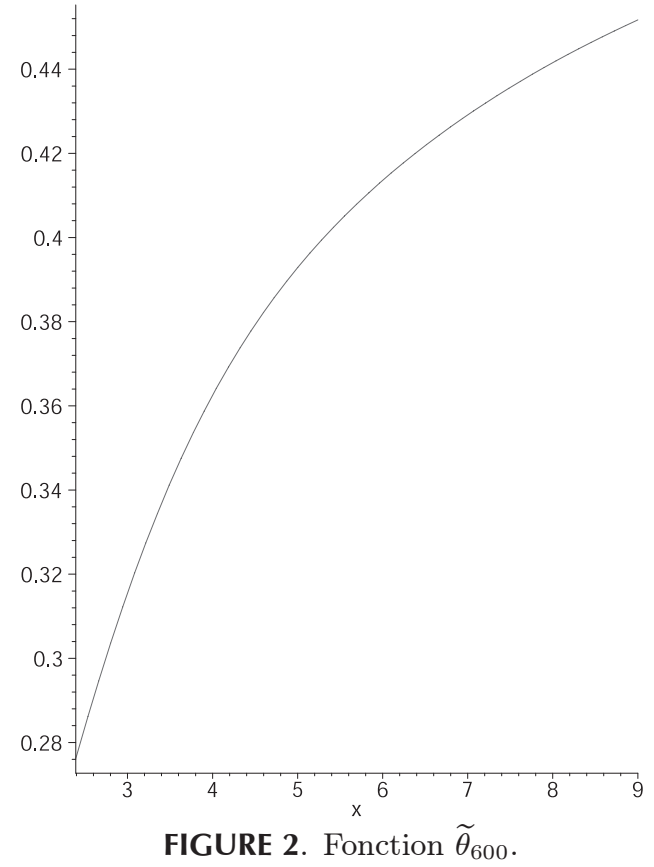

D'autre part après avoir effectué la simplification

$$
T_{N} f_{2} \simeq \sum_{n=1}^{N} \frac{1}{p_{n}} f_{2}\left(\frac{1}{p_{n}}\right) e_{p_{n}, 1},
$$

on a d'après (4-8): $T_{N}^{*} f_{2} \simeq \Gamma_{N}^{*}\left(f_{2}\right)$, où $\Gamma_{N}^{*}\left(f_{2}\right)$ est représentée selon (4-9) par le couple formé du vecteur

$$
\sum_{n=1}^{N} f_{2}\left(\frac{1}{p_{n}}\right) \delta_{p_{n}, 1}
$$

et d'une fonction primitive de

$u \longrightarrow P(M(u))-P(L(N))-\widetilde{\theta}_{N}(u)[P(M(u))-P(m(u))]$.

Ainsi la fonction $\Gamma_{N}^{*} f=\Gamma_{N}^{*} f_{1}+\Gamma_{N}^{*} f_{2}$ approximant $T_{N}^{*} f$ est bien représentée par le couple $(W, \psi)$ de l'énoncé.

Pour déterminer $g_{N}^{(2)}$ nous procédons par approximations successives en calculant

$$
\lim _{n} \Gamma_{N}^{* n} f_{0} /\left\|\Gamma_{N}^{* n} f_{0}\right\|_{1},
$$

où $f_{0}$ est la fonction $\mathbb{1}_{0,2 / 3]}$, c'est-à-dire la fonction représentée selon (4-9) par $\left(\delta_{3,1}, 0\right)$. La convergence est relativement rapide car 20 itérations de $\Gamma_{N}^{*}$ suffisent pour atteindre un résultat stationnaire.

Les calculs avec $N=600$ conduisent à un couple $(V, P)$ représentant $g_{N}^{(2)}$ selon (4-9) vérifiant $P(u) \simeq$ $\lambda \exp u$ pour $u \geq 9$, avec $\lambda=1,28 \ldots$ Comme $L\left(\nu^{-1}(t)\right)=\log \log (1 / t)+o(1)$ et que $\log \left(\nu^{-1}(t)\right)-$

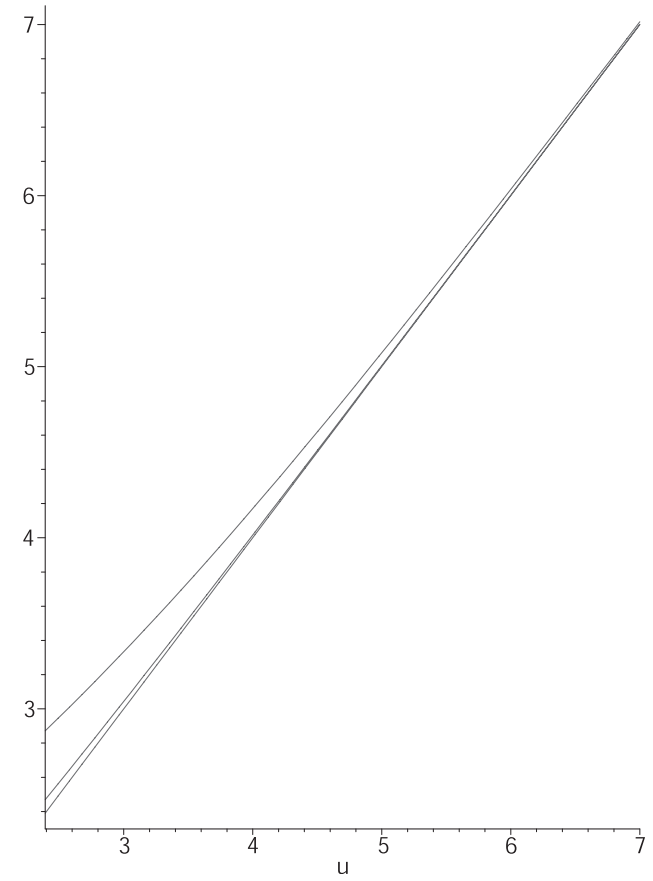

FIGURE 3. Fonctions $u \rightarrow u, u \rightarrow m(u), u \rightarrow M(u)$.

$\log \left(\mu^{-1}(t)\right)=O(\log \log (1 / t))$ on voit que $g_{600}^{(2)}(t) \sim$ $k \log (1 / t)$ avec $k \simeq 1,28$.

En posant $u_{0}=L(600)$, on a $P\left(u_{0}\right) \exp \left(-u_{0}\right) \simeq 0,78$. C'est la valeur de $k$ obtenue par la méthode précédente. On voit que les deux méthodes d'approximation de $\mathrm{g}$ se complètent et ne sont pas contradictoires. Les suites $g_{N}^{(1)}$ et $g_{N}^{(2)}$ semblent bien converger vers la même limite $g$, la deuxième convergeant nettement plus vite que la première, (voir les comparaisons des graphes au $§ 5$ ).

\section{RESULTAT DES CALCULS SOUS MAPLE}

Dans cette partie nous apportons quelques précisions concernant l'algorithme de développement d'un nombre et nous donnons quelques exemples de développement de constantes fondamentales. Puis nous présentons les graphes des approximations de la densité stationnaire $g$ calculées par les deux méthodes exposées au $\S 4$ en les comparant aux histogrammes des orbites issues des constantes précédentes, ceci afin de vérifier via le théorème de Birkhoff l'ergodicité du système.

\subsection{Exemples de développements de nombres irrationnels}

Etant donné un nombre réel $x$, on sait par la proposition 2.14 que son développement d'ordre $n+1$ (noté $\left.S_{n}=\left(p_{0} \ldots p_{n}\right)\right)$ définit une approximation rationnelle 
de $x$ avec une précision inférieure à $1,5 \prod_{i=0}^{n} p_{i}$. En admettant l'hypothèse de l'ergodicité de $\Phi$, la valeur moyenne de $\prod_{i=0}^{n} p_{i}$ est environ égale à $\exp (n E(\log (P))$; où $E(\log (P))$ est l'espérance par rapport à $\mu=g \lambda$ de la fonction $t \rightarrow \log (P(t))$. Or on a $\exp (E(\log (P))=12$ environ. Par conséquent la précision apportée par le développement d'ordre $\mathrm{n}$ d'un nombre est en moyenne $1,5.12^{-n}$. Inversement l'ordre moyen du développement permettant d'obtenir une approximation de $x$ à la précision $10^{-n}$ est approximativement $n \frac{\log (10)}{\log (12)}=0,92 n$.

5.1.1 Description de l'algorithme. Afin d'économiser l'espace mémoire nous déterminons le développement d'un nombre irrationnel $x$ par paquets successifs de longueur pratiquement constante. Pour cela nous nous appuyons sur le fait que si $S_{n}=\left(p_{0} \ldots p_{n}\right)$ est le développement d'ordre $n+1$ de $x$, le reste de son développement coïncide avec celui de la partie fractionnaire de $x \prod_{i=0}^{n} p_{i}$ (voir théorème 2.13).

Voici les articulations de l'algorithme:

Une précision $\varepsilon=10^{-n}$ étant fixée, si $\alpha_{n}$ est l'approximation décimale par défaut de $x$ à $\varepsilon$ près, on sait (voir proposition 2.14) que le développement $S_{k}$ d'ordre $k$ de $\alpha_{n}$ coïncide avec celui de $x$ tant que $\alpha_{n}+\varepsilon$ appartient à $J\left(S_{k}\right)$. Si $k_{n}$ est le maximum des $k$ satisfaisant la propriété précédente, $S_{k_{n}}$ constitue une première liste de longueur environ égale à $0,92 n$. On reprend alors avec la partie fractionnaire de $x \prod_{i=0}^{k_{n}} p_{i}$ et ainsi de suite: si $z$ est le produit des nombres premiers déja déterminés, le paquet suivant s'obtient par le développement de l'approximation à $\varepsilon$ près de la partie fractionnaire de $z x$ en appliquant la même clause d'arrêt. Ce procédé évite de manipuler de trop grands nombres; la part la plus importante du temps de calcul étant prise par la détermination des parties fractionnaires des produits $z x$.

5.1.2 Développement du nombre $\pi-3$. Nous avons déterminé le développement d'ordre 142310 de $\pi$-3, ceci afin d'observer l'apparition d'un premier supérieur à $10^{6}$. Cela se produit pour la première fois au rang 134396 avec le nombre 1508449. Cette attente est relativement longue car le rang moyen du premier superieur à $10^{6}$ dans un développement générique est de l'ordre de 46000 environ (calculé à partir de $g_{600}^{(2)}$ ). Un autre fait surprenant est l'apparition au 66ième rang du nombre 58889; la probabilité de voir apparaître un si grand nombre dans les 66 premiers est inférieure à $2 \%$. Voici le début du développement:
$[11,2,11,5,5,2,5,3,17,11,3,3,11,3,3,11,5,3,23$, 7, 5, 97, 29, 37, 107, 127, 29, 17, 409, 127, 11, 29, 5, 67, $19,43,31,19,103,59,29,7,3,11,11,5,47,29,11,3,5$, $5,3,17,5,29,11,3,3,3,3,5,5,61,151,58889,1877$, $983,757,163,103,79,17,11,2,13]$

Le nombre 58889 apparait une seconde fois au 125706ième rang. Voici l'extrait du développement concerné:

$[23,11,5,5,149,53,43,27103,2281,58889,23039,3037$, $347,83,23,163,37,7,79,19,17,5,5,3,17,5,149,31$, $37,7,5,17,37,7,17,13,83,23,19,37,7,37,127,17$, $5,3,3,7,5,5,3,5,2,5,5,2]$

Voici l'extrait du développement où apparaît 1508449:

$[7,7,53,29,5,7,17,7,3,7,5,37,7,23,13,23,11$, $11,3,3,11,7,7,7,7,7,3,29,7,5,29,1508449,53407$, 10037, 389, 67, 29, 11, 2, 43, 37, 13, 137, 61, 47, 89, 19, $17,5,7,5,17,5,5,11,2]$

Voici rangée dans l'ordre croissant la liste (avec répétition) des plus grands nombres de ce développement:

[9767, 9803, 10037, 10037, 10061, 10061, 10061, 10169, 10177, 10211, 10331, 10391, 10391, 10427, 10589, 10601, 10739, 10831, 10903, 10937, 10957, 10973, 11069, 11239, $11251,11423,11587,11617,11777,11801,11863,11863$, 12211, 12227, 12473, 12497, 12637, 12653, 12757, 12809, 12821, 12823, 13033, 13249, 13553, 13613, 13649, 13669, 13721, 13751, 14057, 14281, 14369, 14771, 14879, 15013, 15031, 15101, 15187, 15307, 15391, 15493, 15641, 15887, 15901, 16127, 16127, 16267, 16301, 16349, 16519, 16691, 16811, 16921, 17377, 17597, 17597, 18119, 18251, 18301, 18583, 18593, 18911, 19009, 19069, 19211, 19469, 19489, 19661, 19961, 19961, 19973, 20849, 20849, 21169, 21191, 21247, 21401, 21599, 21751, 22247, 22277, 22291, 22303, 22637, 22669, 22807, 23039, 23909, 24197, 24953, 25031, 25219, 25793, 26107, 26141, 26399, 27059, 27103, 27611, 27647, 27743, 27917, 28027, 28387, 29017, 29363, 29641, 30071, 30241, 30403, 30427, 31601, 32297, 32687, 32779, 33317, 33487, 36493, 37619, 38011, 39133, 39503, 40087, 40387, 40529, 41351, 42101, 42443, 42487, 43189, 44867, 46171, 46261, 47857, 47911, 48731, 49843, 50207, 53407, 54139, 54941, 58477, 58889, 58889, 59207, 60493, 60821, 61297, 61487, 62927, 66553, 67103, 68729, 69481, 76667, $78607,80447,83389,86923,87337,98867,102139$, 102407, 107071, 109919, 127781, 143827, 153871, 167861, 206233, 271357, 294179, 321467, 326219, 326737, 334379, 398569, 817123, 900539, 1508449]

5.1.3 Développement du nombre $e-2$. Ce développement présente la particularité de produire 
relativement tôt un nombre supérieur à $10^{7}$, à savoir 30225161 au rang 11063.

Voici l'extrait du développement où ce nombre apparait:

$[5,3,17,7,5,31,19,373,2203,131,89,17,11,2,37,11$, $2,157,37,11,3,3,3,7,67,163,31,331,6547,15373$, 681259, 1540477, 30225161, 17403227, 636263, 1454347, 820399, 25679, 10831, 1381, 223, 37, 7, 5, 5, 53, 53, 787, $103,149,191,29,11,17]$

Voici rangée dans l'ordre croissant la liste des plus grand nombres du développement d'ordre 11972 de $e-2$ :

[3613, 3907, 3989, 3989, 4027, 4211, 4507, 4861, 5009, 5113, 5209, 5227, 5417, 5897, 5903, 6547, 6947, 7297, 7369, 7649, 8147, 8609, 8693, 9151, 9257, 9463, 9533, 10831, 12889, 12889, 14243, 14537, 15217, 15373, 18127, 19949, 24631, 25537, 25679, 26879, 69467, 89041, 106781, 202049, 636263, 681259, 820399, 1454347, 1540477, 17403227, 30225161].

5.1.4 Développement de $\sqrt{2}-1$. Bien que l'on observe ici relativement tôt l'apparition d'un grand nombre (842321) au 18989ième rang, il faut attendre le 63626ième rang avec 3355487 pour dépasser $10^{6}$. Et presque aussitôt après apparaît le nombre 24581083.

Voici l'extrait concerné:

$[3,5,2,29,11,3,7,37,11,2,29,17,5,3,11,149,19$, 17, 11, 2, 3355487, 240967, 31963, 39079, 41729, 4889, $2887,1511,211,37,127,19,37,19,23,7,7,5,2,5,5$, $67,239,67,83,47,17,5,29,11,5,2,13,7,7,5,5,3$, $3,7,5,5,113,31,23,17,5,3,3,3,3,23,7,17,23,5$, $37,29,5,5,7,11,3,3,11,3,7,29,31,23,17,5,3,7$, $5,3,11,11,5,3,59,11,11,5,2,5,5,53,37,53,11,11$, $11,2,11,223,53,29,37,1187,727,2251,331,31,29,5$, $5,79,23,29,11,2,11,5,2,5,2,5,7,3229,24581083$, 1664987, 3730033, 432569, 67987, 15823, 64187, 14489, $5953,2357,439,149,17,11,19]$

Voici les plus grands nombres:

[22783, 23399, 26203, 27031, 27581, 28283, 29683, 29717, 30241, 31963, 33247, 34313, 34403, 34457, 34667, 35831, 36373, 38933, 39079, 41729, 44159, 52067, 55619, 57587, 59651, 61331, 61751, 62141, 64187, 67073, 67987, 85009, 98711, 107941, 113497, 120811, 134581, 135277, 144289, 148639, 176041, 180797, 220973, 240967, 338803, 432569, 842321, 1664987, 3355487, 3730033, 24581083].

5.1.5 Développement de $\pi^{2}$-9. Le rang du premier supérieur à $10^{6}$ ( 2411821) est 12374 .

\section{Voici l'extrait concerné:}

$[5,5,3,79,17,11,31,181,233,97,311,2411821$, 1046447, 43987, 4943, 1171, 787, 79, 67, 11, 19, 47, 13, $23,7,5,17,5,29,5,29,11,7,3,11,5,2,5,5,5,29,5$, $11,7,11,5]$

Voici les plus grands nombres:

$[4567,4643,4751,4861,4943,5381,5387,5897,6079$, 6101, 6143, 6373, 6521, 6761, 6803, 7103, 7207, 7219, 7321, 7789, 7817, 7853, 8243, 8951, 9767, 9941, 10289, 10477, 11299, 12197, 12889, 13421, 14591, 17939, 20287, 21157, 21893, 22769, 24989, 29059, 30089, 32507, 35933, 36479, 43987, 52769, 64373, 68729, 76913, 1046447, 2411821]

5.1.6 Développement du nombre $(\sqrt{5}-1) / 2$. Les plus grands nombres du developpement d'ordre 103706:

[38011, 38197, 39839, 40627, 41047, 44371, 44579, 44617, 44983, 46021, 46091, 46261, 46663, 47591, 49801, 51001, 53087, 53993, 55229, 59729, 61001, 63719, 65921, 66683, 67939, 68863, 69767, 74071, 75431, 76819, 77417, 79133, 82531, 84481, 91453, 95737, 98443, 100447, 102101, 106163, 149993, 160309, 171271, 218599, 227453, 229979, 253381, 292021, 300277, 377021, 652903]

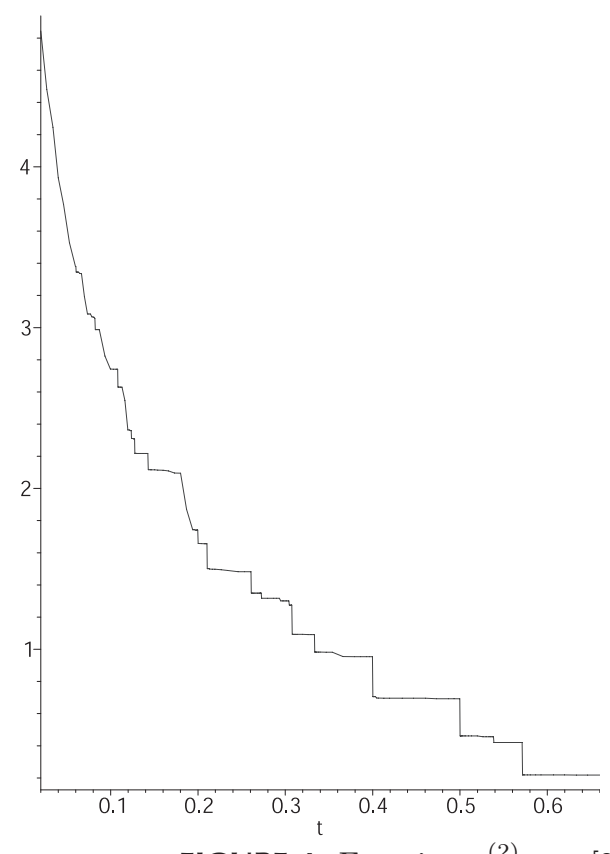

FIGURE 4. Fonction $g_{600}^{(2)}$ sur $[0.02,1.5]$.

\subsection{Calcul de la densité stationnaire et des coefficients qui en dépendent}

Commençons par apporter quelques précisions concernant la programmation. Les fonctions intervenant dans 


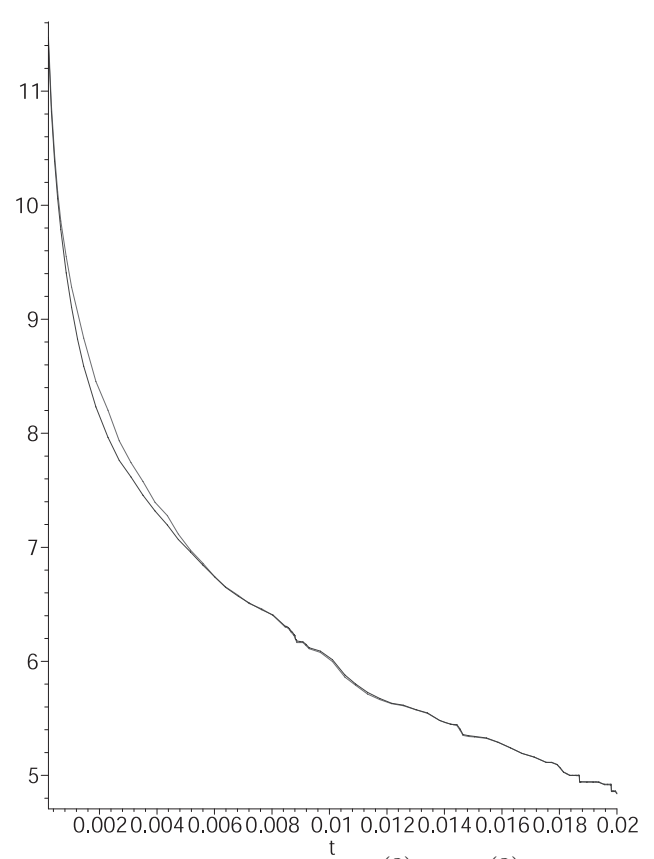

FIGURE 5. Fonctions $g_{600}^{(2)}$ et $g_{200}^{(2)}$ sur $[0.0002,0.02]$ (sur $[0.02,1.5]$ on observe une presque parfaite superposition des deux graphes).

l'expression de l'opérateur $\Gamma_{N}^{*}$ (voir $\left.\oint 4\right)$ sont rentrées dans les programmes sous forme de spline linéaire.

Tout se passe alors avec des fonctions polynomiales par morceaux. Pour éviter les saturations il est nécessaire d'introduire des procédures de calcul explicite de la liste des noeuds des fonctions composées.

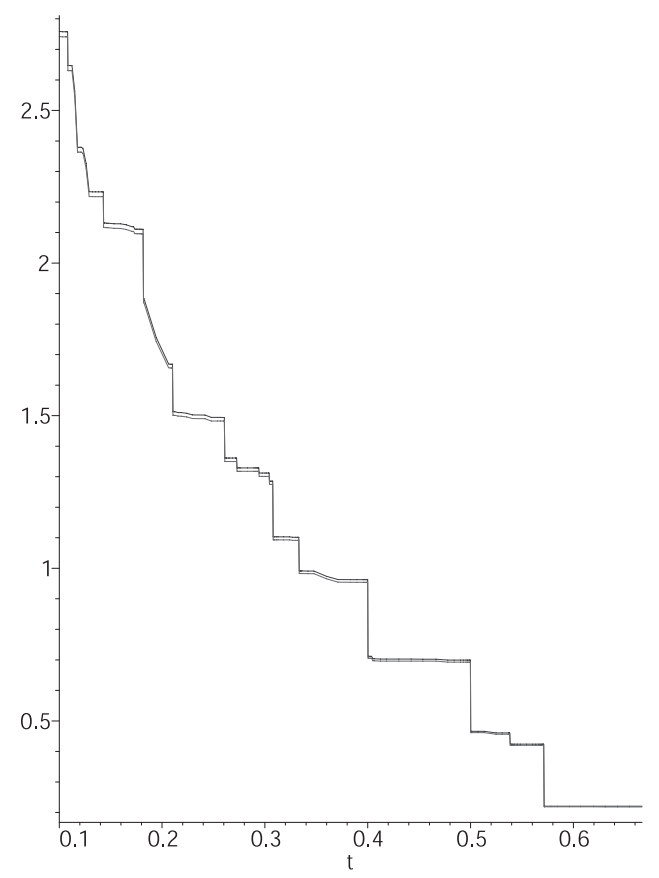

FIGURE 6. Fonctions $g_{600}^{(2)}$ et $g_{600}^{(1)}$ sur $[0.1,1.5]$.

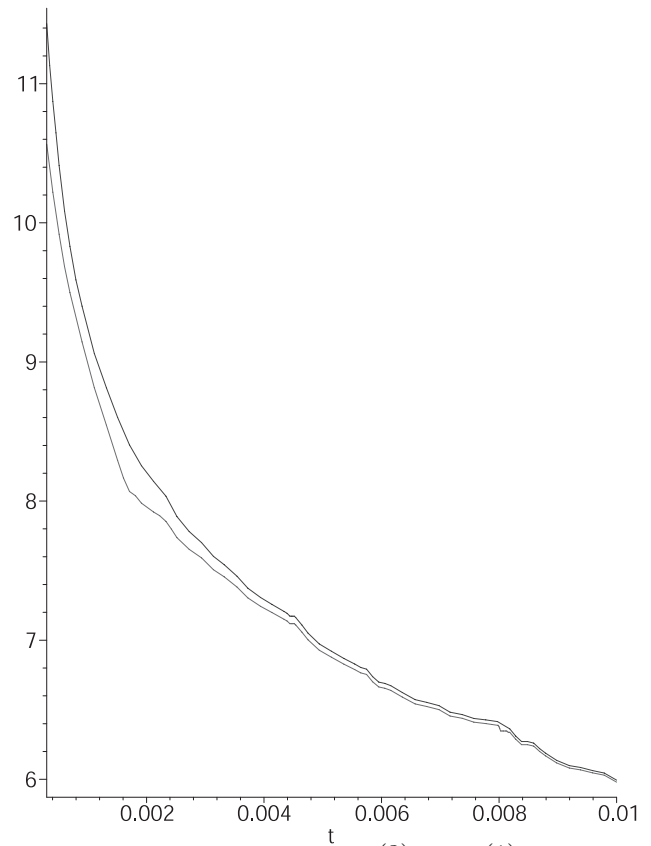

FIGURE 7. Fonctions $g_{600}^{(2)}$ et $g_{600}^{(1)}$ sur [0.0003,0.01]. On voit l'effet régularisant de la seconde méthode. La jonction entre les parties étagées et continues des approximations de $\mathrm{g}$ (formules (4-3) et (4-9)) se fait de manière plus progressive pour $g_{600}^{(2)}$.

D'autre part afin d'alléger les calculs, à chaque itération de l'opérateur, seules les $\mathrm{N}$ composantes d'indices $\left(p_{n}, 1\right)$ du produit matriciel $\mathcal{A}_{N} V$ sont effectivement déterminées, les autres composantes sont complétées en accord avec les relations du $§ 3.3$.

\subsection{Comparaison du graphe de la densité stationnaire avec les histogammes des orbites issues de certains nombres}

A l'occasion du calcul du développement des nombres $\pi-3, \sqrt{2}-1,(\sqrt{5}-1) / 2$ nous avons déterminé la liste des moyennes temporelles de $\Phi^{n}(x)$ pour les intervalles $[k / 1000,(k+1) / 1000](0<k<20),[k / 100,(k+1) / 100]$ $(1<k<66)$ et $[66 / 100,2 / 3]$. La concordance observée entre les histogrammes construits à partir de ces listes et le graphe de $g_{600}^{(2)}$ montre l'efficacité de la seconde méthode d'approximation de $g$ et confirme l'hypothèse d'ergodicité de $\Phi$.

\subsection{Paramètres associés à $g$}

Liste des dix premiers $\alpha_{p, 1}$.

calculée à partir de $g_{200}^{(2)}$ :

$[2,0.0364][3,0.11562][5,0.14578][7,0.09960][11$, $0.11531][13,0.04095]$ [17 0.05587] [19, 0.021406$]$ [23, $0.03258][29,0.03453]$ 
Experimental Mathematics, Vol. 11 (2002), No. 3

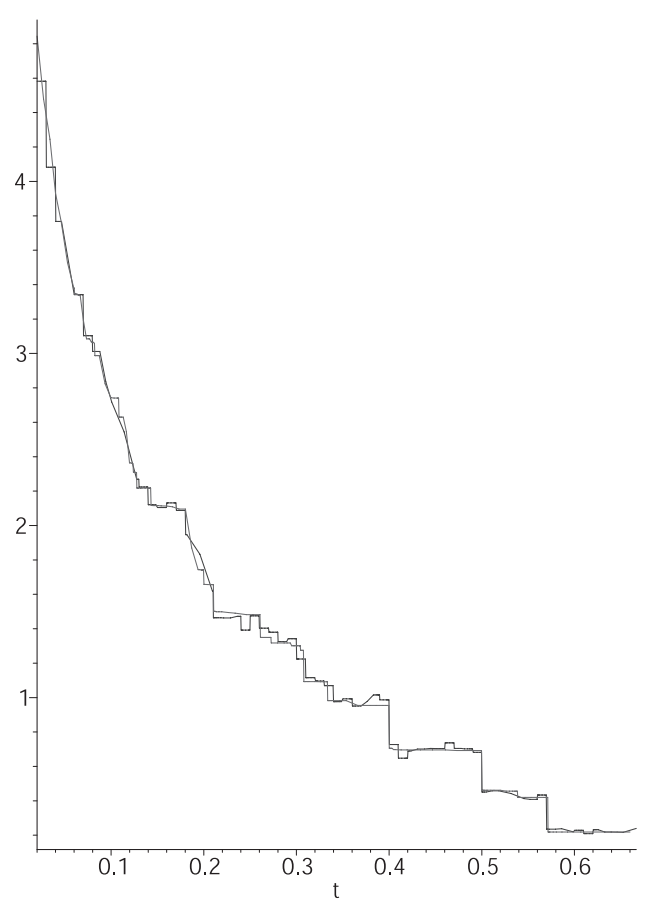

FIGURE 8. Histogramme de l'orbite de $\pi-3$ et $g_{600}^{(2)}$ sur $[0.02,1.5]$.

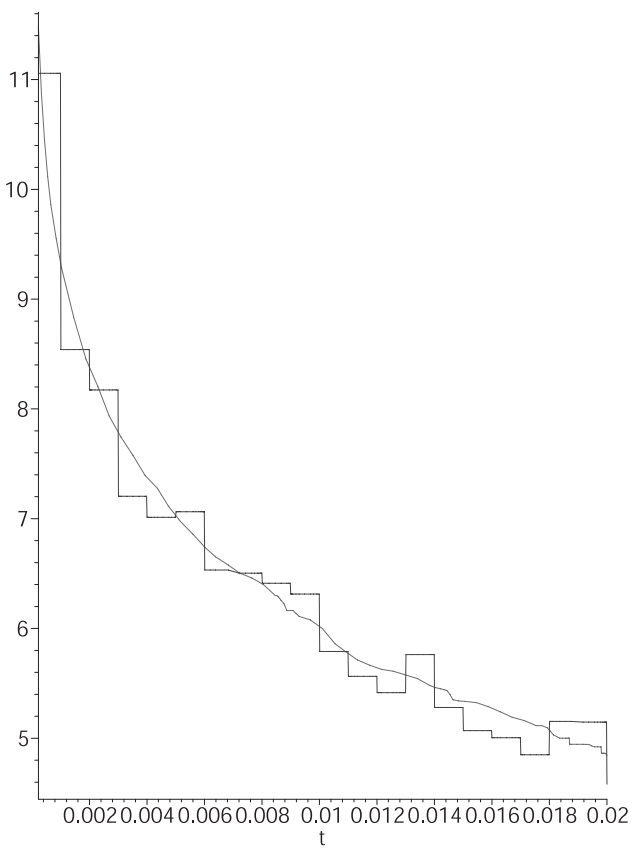

FIGURE 9. Histogramme de l'orbite de $\pi-3$ et $g_{600}^{(2)}$ sur $[0,0.02]$.

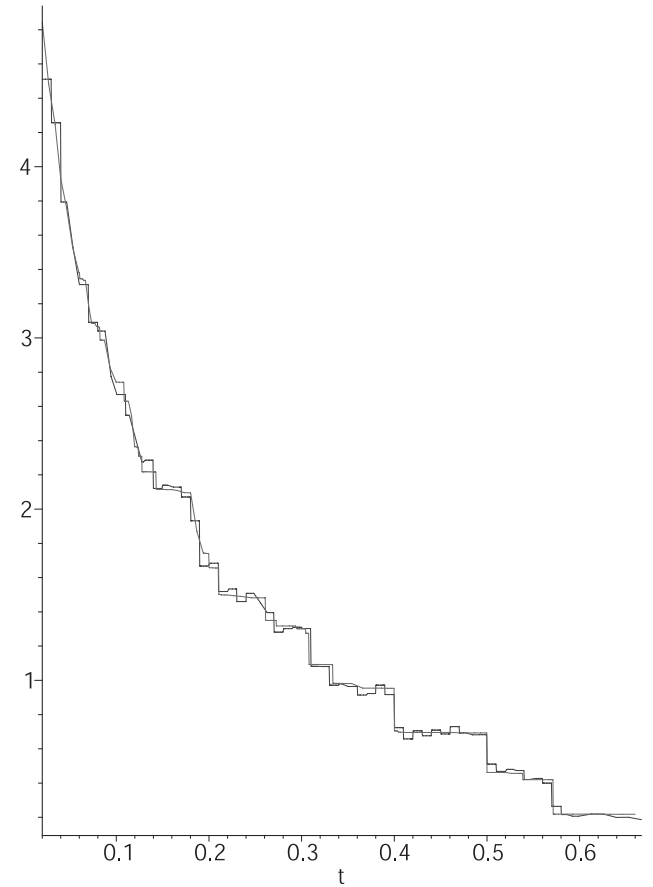

FIGURE 10. Histogramme de l'orbite de $(\sqrt{5}-1) / 2$ et $g_{600}^{(2)} \operatorname{sur}[0.02,1.5]$.

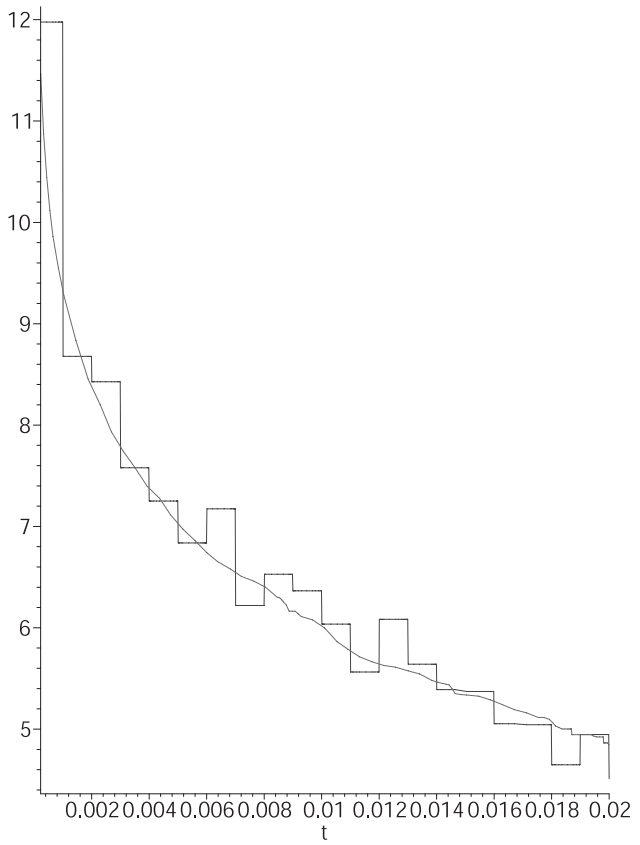

FIGURE 11. Histogramme de l'orbite de $(\sqrt{5}-1) / 2$ et $g_{600}^{(2)}$ sur [0, 0.02]. 
calculée à partir de $g_{600}^{(2)}$ :

$[2,0.03638][3,0.11544][5,0.14556][7,0.09948][11$, $0.11518][13,0.04092][17,0.05582][19,0.02139]$ [23, $0.03256][29,0.03452]$

Liste des dix premiers coefficients de la loi de la variable $\mathrm{P}$ (c'est à dire des probabilités d'apparition des dix premiers nombres premiers)

calculée à partir de $g_{200}^{(2)}$ :

$[2,0.0524][3,0.1340][5,0.1824][7,0.1154][11,0.1299]$

$[13,0.0421][17,0.0589][19,0.0216][23,0.0339][29$, $0.0360]$

calculée à partir de $g_{600}^{(2)}$ :

$[2,0.0523][3,0.1338][5,0.1822][7,0.1152][11,0.1298]$ $[13,0.0421][17,0.0588][19,0.0216][23,0.0339][29$, $0.0359]$

Limite en 0 de $\mathrm{g}(\mathrm{t}) / \log (1 / \mathrm{t})$ :

avec $g_{200}^{(2)}: 1,19$, avec $g_{600}^{(2)}: 1,28$

Liste pour $\mathrm{n}$ de 5 à 9 des rangs moyens des premières apparitions d'un nombre supérieur à $10^{n}$ calculée à partir de $g_{200}^{(2)}$ :

[5645, 47025, 402673, 3489183, 28490028] calculée à partir de $g_{600}^{(2)}$ :

[5610, 46622, 398311, 3456619, 29239766]

\section{REMERCIEMENTS}

Je remercie Christian Ballot de m'avoir suggéré d'introduire la notion d'indice de bifurcation afin de clarifier l'exposé.

\section{BIBLIOGRAPHIE}

[Cramér 36] H. Cramér. "On the order of magnitude of the difference between consecutive primes." Acta. Arith. 2 (1936), 396-403.

[Ellison et Mendès France 75] W. J. Ellison et M. Mendès France. Les nombres premiers Hermann, Paris, 1975.

[Feller 68] W. Feller. An introduction to Probability Theory and Its Applications, Vol. I, John Wiley and Sons, New York, 1968.

[Lasota and Mackey 94] A. Lasota and M. C. Mackey. Chaos, Fractals, and Noise, Springer, Berlin-Heidelberg-New York, 1994.

[Schweiger 95] F. Schweiger.Ergodic theory of fibered systems and metric number theory, Oxford Press, London, 1995.

[Ulam 60] S. M. Ulam. A collection of Mathematical problems, Interscience Tracts in Pure and Applied Math., vol. 8, Interscience, New York, 1960.

Alain Costé, LMNO, B.P. 5186, Université campus 2, 14032 Caen cedex, France (Coste@math.unicaen.fr)

Received April 30, 2001; accepted in revised form October 10, 2001. 